\title{
Expanded Cyclotetrabenzoins
}

Andrew M. Eisterhold, ${ }^{a}$ Thamon Puangsamlee, ${ }^{a}$ Steffen Otterbach, ${ }^{b}$ Stefan Bräse, ${ }^{b, c}$ Patrick Weis, ${ }^{d}$ Xiqu Wang, ${ }^{a}$ Ksenia V. Kutonova, ${ }^{b, *}$ and Ognjen Š. Miljanića,*

${ }^{a}$ University of Houston, Department of Chemistry, 3585 Cullen Boulevard \#112, Houston, TX 77204-5003, United States

${ }^{\mathrm{b}}$ Institute of Organic Chemistry, Karlsruhe Institute of Technology (KIT), Fritz-Haber Weg 6, 76131 Karlsruhe, Germany

${ }^{c}$ Institute of Biological and Chemical Systems, Karlsruhe Institute of Technology (KIT), Hermann-von-Helmholtz-Platz 1, 76344 Eggenstein-Leopoldshafen, Germany

${ }^{\mathrm{d}}$ Institute of Physical Chemistry, Karlsruhe Institute of Technology (KIT), Fritz-Haber Weg 2, 76131 Karlsruhe, Germany

Email: ksenia.kutonova@kit.edu,miljanic@uh.edu

Phone: +1.832 .842 .8827$

\section{Supporting Information}

General Methods and Materials. . .52

Possible Regio- and Stereoisomers of $\mathbf{2 a}$..................................................................................................................

Synthesis of Cyclotetrabenzoin 2a Using an NHC Catalyst..........................................................................S5

Synthesis of Cyclotetrabenzoin $\mathbf{5 b}$.........................................................................................................................

Synthesis of 4,4'-(ethyne-1,2-diyl) dibenzaldehyde 1c ...............................................................................S7

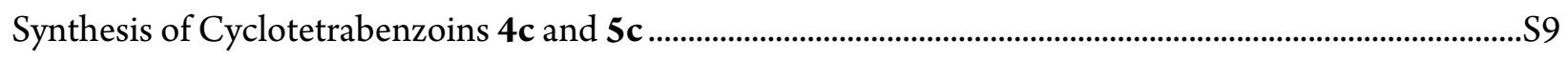

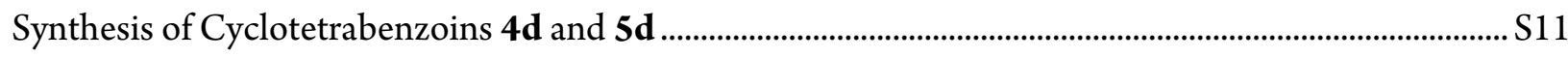

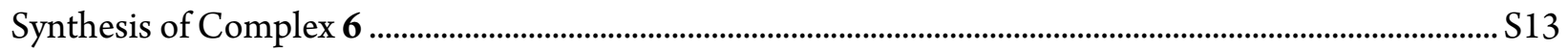

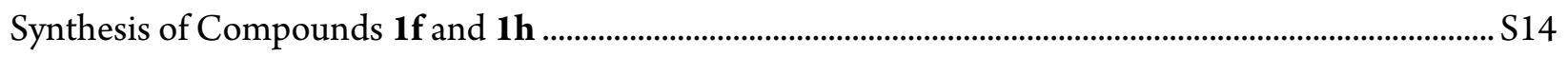

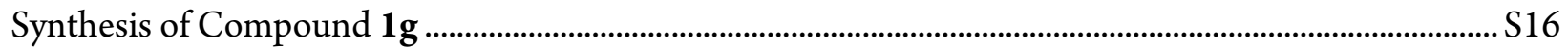

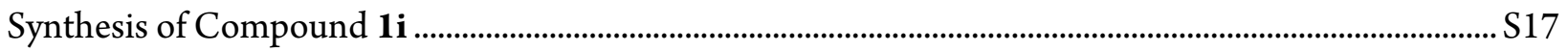

Crystal Data and Structure Refinement Parameters for Compound 5b ........................................................ S19

Crystal Data and Structure Refinement Parameters for Compound 4d....................................................... S21

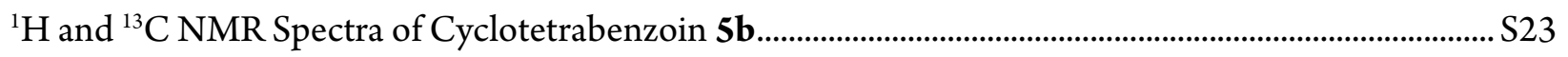

${ }^{1} \mathrm{H}$ and ${ }^{13} \mathrm{C}$ NMR Spectra of Cyclotetrabenzoins 4c and 5c.......................................................................... S24

${ }^{1} \mathrm{H}$ and ${ }^{13} \mathrm{C}$ NMR Spectra of Cyclotetrabenzoin 4d and 5d ....................................................................... S26

${ }^{1} \mathrm{H},{ }^{13} \mathrm{C}$ NMR, IR, and HR-ESI Mass Spectra of Complex 6 ......................................................................... S28

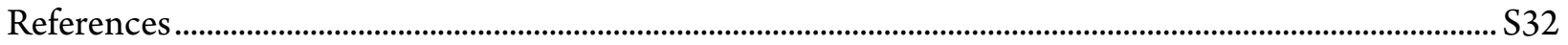




\section{General Methods and Materials}

All reactions were performed under inert atmosphere in oven-dried glassware. Reagents were purchased from commercial suppliers: 3-ethyl-5-(2-hydroxylethyl)-4-methylthiazolium bromide (Thermo-Fischer Scientific), 2,6-naphthalenedicarboxylic Acid (TCI), triethylamine (Thermo-Fischer Scientific), $\mathrm{Ac}_{2} \mathrm{O}$ (Sigma-Aldrich), $\mathrm{LiAlH}_{4}$ and $\mathrm{H}_{2} \mathrm{SO}_{4}$ (Macron Fine Chemicals) and used without further purification, except for 4,4'-biphenyldicarbaldehyde, which was purified by recrystallization from EtOH. Solvents were used as received, except $\mathrm{PhMe}$, which was dried over activated alumina in an mBraun solvent purification system. Compound 2,7-naphthalenedialdehyde was prepared according to the literature procedure. ${ }^{1}$

Column chromatography was carried out on silica gel 60, 32-63 mesh. Analytical TLC was performed on J. T. Baker or Merck plastic-backed silica gel IB-F plates. NMR spectra were obtained on Bruker Avance 300, Bruker Avance 400, Bruker Avance DRX 500, JEOL ECA-500, and ECA-600 spectrometers, with working frequencies (for ${ }^{1} \mathrm{H}$ NMR nuclei) of 300, 400, 500, 500, and $600 \mathrm{MHz}$, respectively. All ${ }^{13} \mathrm{C}$ NMR spectra were recorded with simultaneous decoupling of ${ }^{1} \mathrm{H}$ nuclei. ${ }^{1} \mathrm{H}$ NMR chemical shifts are reported in ppm units relative to the residual signal of the solvent $\left(\mathrm{CDCl}_{3}: 7.26 \mathrm{ppm}\right.$, DMSO- $\left.d_{6}: 2.50 \mathrm{ppm}\right)$. All NMR spectra were recorded at $25^{\circ} \mathrm{C}$.

Mass spectrometric measurements were performed by the Mass Spectrometry Facility of the Department of Chemistry and Biochemistry at the University of Texas at Austin. Mass spectra of aldehydes 1c, 5a-5c, and $\mathbf{6}$ and corresponding intermediate compounds were recorded on a Finnigan MAT $95(70 \mathrm{eV})$ using EIMS (electron ionization mass spectrometry) or FAB-MS (fast atom bombardment mass spectrometry) with 3-nitrobenzyl alcohol as a matrix. Mass spectra of 3c was recorded on ThermoFisher QExactive Plus using ESI MS (electrospray ionization mass spectrometry).

The molecule ion peak $[\mathrm{M}]^{+}$or peak of the pseudo molecule $[\mathrm{M}+\mathrm{H}]^{+}$are indicated as mass per charge ratio $(\mathrm{m} / \mathrm{z})$ and their intensity is given in percent, relative to the main peak (100\%) for EIMS. Structure of 7 was solved as iodide adduct $[\mathrm{M}+\mathrm{I}]^{-}$by HR-ESI MS (high resolution electrospray ionization mass spectrometry) using a Thermo Scientific LTQ Orbitrap XL at KIT. The measurements were performed in negative mode with a $\mathrm{CH}_{2} \mathrm{Cl}_{2}: \mathrm{MeOH}(1: 1)$ solution of 7 with $1 \% 1 \mathrm{mM}$ solution of $\mathrm{CsI}$ in $\mathrm{MeOH}$ added. Collision induced dissociation $(\mathrm{CID})$ of $[\mathrm{M}+\mathrm{I}]^{-}$indicates sequential loss of up to $24 \mathrm{CO}$ 
molecules. Infrared spectra were recorded on either Nicolet iS10 FT-IR or Bruker IFS 88 spectrometer; both were equipped with an ATR probe. The UV-vis absorption spectra were measured using a Cary 5000 UV-Vis-NIR spectrophotometer from Agilent Technology. The measurement was performed for the $8.9 \mu \mathrm{M}$ solutions in $\mathrm{CH}_{2} \mathrm{Cl}_{2}$ as a solvent in the range from $700 \mathrm{~nm}$ to $220 \mathrm{~nm}$ and using the cuvettes with a thickness of $1 \mathrm{~cm}$. Melting points were measured in open capillary tubes using either a Barnstead International Mel-TEMP apparatus or Stanford Research Systems Optimelt and are uncorrected.

All single X-ray diffusion measurements were performed by Dr. Xiqu Wang (UH) by using a Bruker DUO platform diffractometer equipped with a 4K CCD APEX II detector and an Incoatec 30 Watt Cu microsource with compact multilayer optics. Data were collected using a narrow-frame algorithm with scan widths of $0.50 \%$ in omega and an exposure time of $20 \mathrm{~s}$ per frame at $4 \mathrm{~cm}$ detector distance. The data were integrated using the Bruker SAINT program, with the intensities corrected for Lorentz factor, polarization, air absorption, and absorption due to the variation in the path length through the detector faceplate. The data were scaled, and an absorption correction was applied using SADABS. The structure was solved with SHELXT 2014, and refined with SHELXL 2014 using full-matrix least-squares refinement. The non- $\mathrm{H}$ atoms were refined with anisotropic thermal parameters, and all the $\mathrm{H}$ atoms were calculated in idealized positions and refined riding on their parent atoms. The highly disordered solvent molecules could not be determined reliably and their contributions to the electron density were treated using the PLATON/SQUEEZE program. The chemical formulas and calculated density correspond to the ordered molecules only.

Gas adsorption isotherms were measured volumetrically using a surface analyser ThermoScientific Surfer Gas adsorption Porosimeter. A liquid nitrogen bath $(77 \mathrm{~K})$ was used and the $\mathrm{N}_{2}$ gas used was UHP grade. For measurement of the specific surface areas $\left(S_{\mathrm{BET}}, \mathrm{m}^{2} / \mathrm{g}\right)$ the BET method was applied. None of the examined samples showed any porosity.

Experiments are presented in the order that follows the discussion of the manuscript. Compound numbers are identical to those in the main text of the manuscript. 


\section{Possible Regio- and Stereoisomers of 2a}

Figure S1. Possible regio- and stereosiomers of cyclotetrabenzoin 2a. Isomers highlighted in red are chiral and therefore exist as two enantiomers.
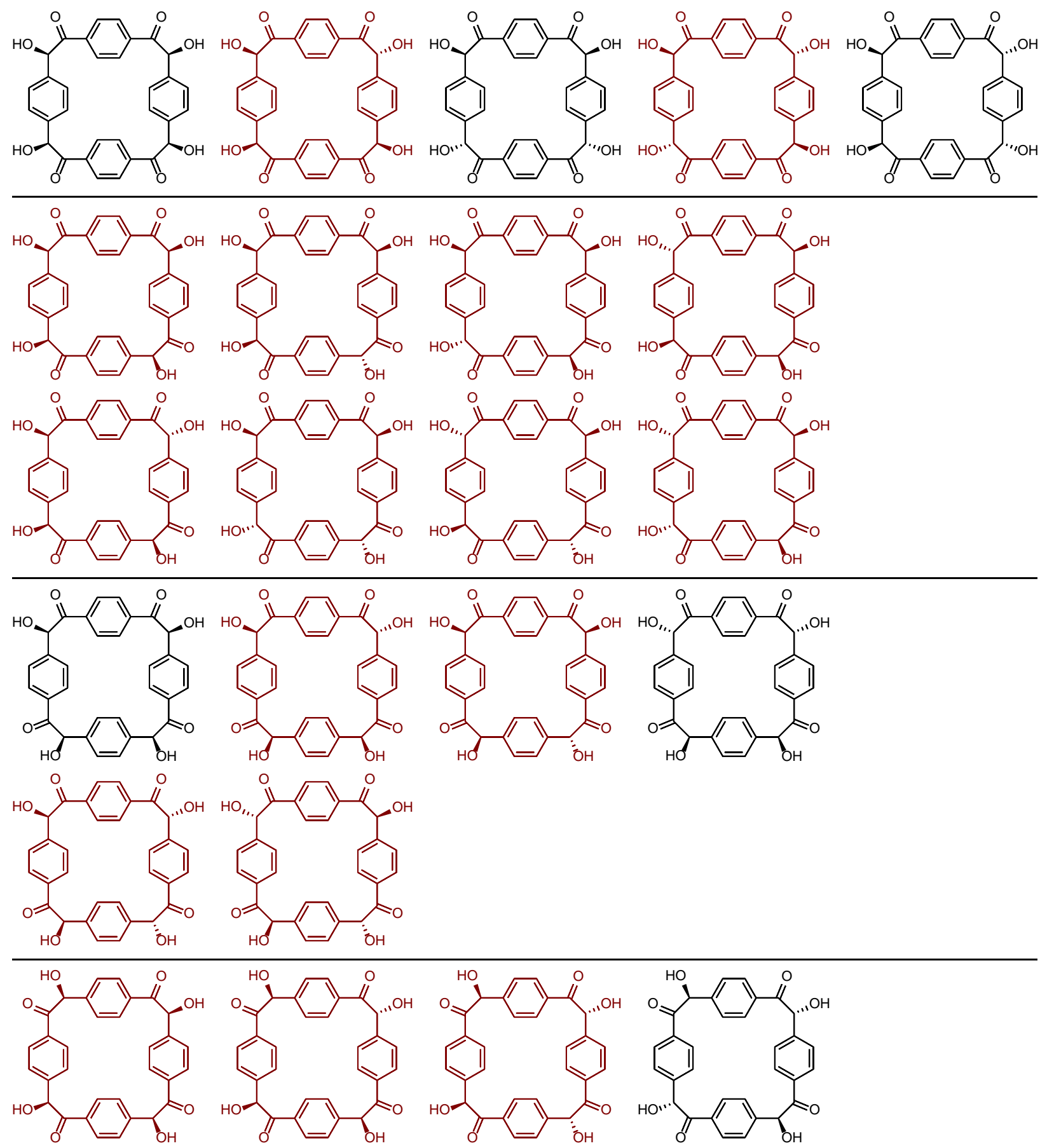


\section{Synthesis of Cyclotetrabenzoin 2a Using an NHC Catalyst}

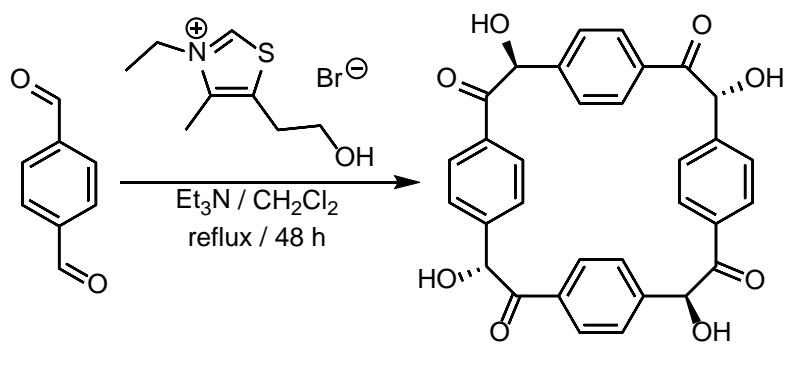

$1 \mathbf{a}$

$2 a$

Terephthalaldehyde (1a, $134 \mathrm{mg}, 1.00 \mathrm{mmol})$ and 3-ethyl-5-(2-hydroxyethyl)-4-methyl thiazolium bromide (50 mg, $0.199 \mathrm{mmol}$ ) were dissolved in $\mathrm{CH}_{2} \mathrm{Cl}_{2}(40 \mathrm{~mL})$, and the solution was then heated to boiling in an oil bath. Then, $\mathrm{Et}_{3} \mathrm{~N}(0.1 \mathrm{~mL})$ was added and the solution was heated at reflux for $72 \mathrm{~h}$. The resulting precipitate was filtered, washed three times with $\mathrm{CH}_{2} \mathrm{Cl}_{2}(25 \mathrm{~mL})$ and dried. Cyclotetrabenzoin $\mathbf{2 a}$ was isolated from the resulting solid by recrystallization from DMSO and $\mathrm{MeOH}$ (25 mg, 19\% yield), following the procedure previously developed to purify $\mathbf{2 a}$ obtained in the cyanidecatalyzed reaction. ${ }^{2}$ The recrystallized material was proven to be identical (by ${ }^{1} \mathrm{H}$ NMR spectroscopy) to the one prepared in the cyanide-catalyzed reaction. 


\section{Synthesis of Cyclotetrabenzoin $5 b$}

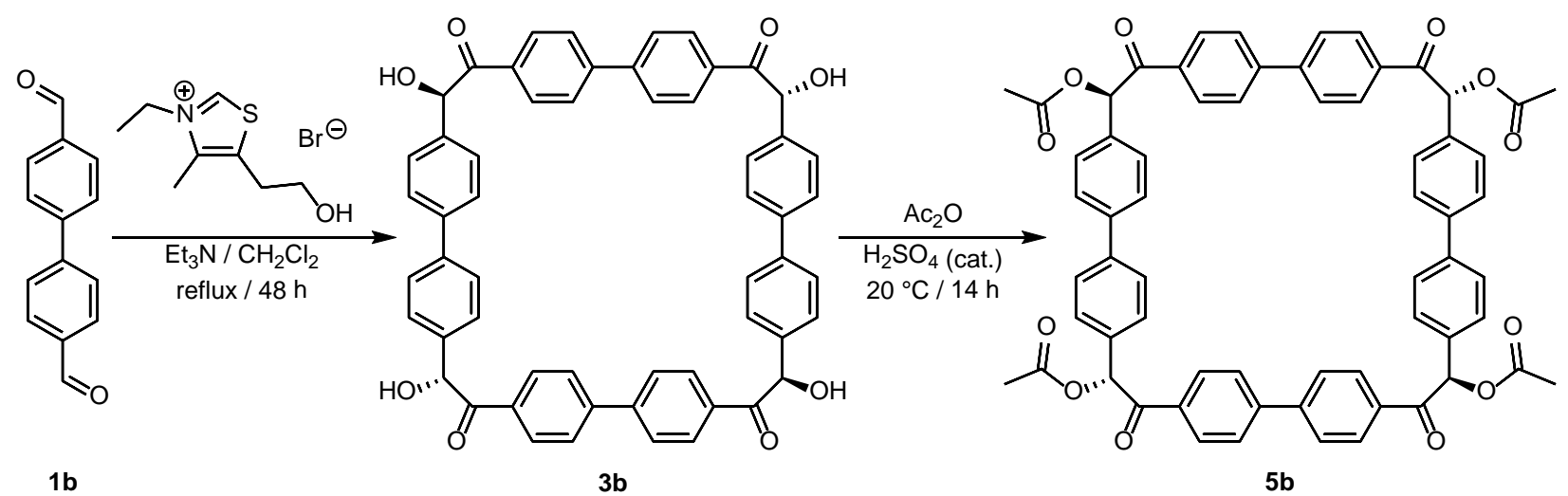

A solution of 4,4'-biphenyldicarbaldehyde (1 b, $2.11 \mathrm{~g}, 10.0 \mathrm{mmol})$ and 3-ethyl-5-(2-hydroxyethyl)-

4-methylthiazolium bromide (500 mg, $1.98 \mathrm{mmol}$ ) in $\mathrm{CH}_{2} \mathrm{Cl}_{2}(40 \mathrm{~mL})$ was heated to reflux (oil bath) under a nitrogen atmosphere. Then, $\mathrm{Et}_{3} \mathrm{~N}(2.00 \mathrm{~mL}, 1.45 \mathrm{~g}, 14.3 \mathrm{mmol})$ was injected and the reaction mixture was kept at reflux for $48 \mathrm{~h}$. While still hot, the solution was filtered, and the residue was washed with $\mathrm{CH}_{2} \mathrm{Cl}_{2}, \mathrm{EtOH}$, and $\mathrm{Et}_{2} \mathrm{O}$. After drying, $\sim 1.19 \mathrm{~g}$ of crude product $\mathbf{3 b}$ was obtained as a white to beige powder ( $\mathrm{mp}>300^{\circ} \mathrm{C}$, decomp. $)$. IR: $\tilde{v}=3400,1680 \mathrm{~cm}^{-1}$.

Crude biphenyl cyclotetrabenzoin $(3 \mathrm{~b}, 1.19 \mathrm{~g}, 1.42 \mathrm{mmol})$ was stirred in $\mathrm{Ac}_{2} \mathrm{O}(8.0 \mathrm{~mL}, 8.64 \mathrm{~g}, 84.5$ mmol) until the mixture turned homogeneous. A catalytic amount of $\mathrm{H}_{2} \mathrm{SO}_{4}$ (4 drops) was added and the mixture was stirred for $18 \mathrm{~h}$ at room temperature. Then, the mixture was neutralized with $1 \mathrm{M}$ solution of $\mathrm{NaOH}(3 \mathrm{~mL})$. The mixture was poured into $\mathrm{CH}_{2} \mathrm{Cl}_{2}(100 \mathrm{~mL})$, transferred to a separatory funnel, and washed with deionized $\mathrm{H}_{2} \mathrm{O}(100 \mathrm{~mL})$. The organic layer was removed via rotary evaporator to provide a yellow, glassy solid. The crude product was purified by column chromatography on silica gel, eluting with an 8:2 EtOAc/hexanes mixture. After evaporation, compound $\mathbf{5 b}$ (622 mg, $0.62 \mathrm{mmol}$, $24 \%$ overall yield) was collected as a white solid (mp $>300{ }^{\circ} \mathrm{C}$, decomp.). ${ }^{1} \mathrm{H} \mathrm{NMR}\left(600 \mathrm{MHz}, \mathrm{CDCl}_{3}\right)$ : $\delta 8.06-7.85(\mathrm{~m}, 8 \mathrm{H}), 7.63-7.42(\mathrm{~m}, 24 \mathrm{H}), 6.95-6.80(\mathrm{~m}, 4 \mathrm{H}), 2.23(\mathrm{~s}, 12 \mathrm{H}) \mathrm{ppm} .{ }^{13} \mathrm{C}$ NMR $(150$ $\mathrm{MHz}, \mathrm{CDCl}_{3}$ ): $\delta$ 192.9-192.4 (m), 170.6, 170.4, 168.9, 144.9-144.7 (m), 144.2-144.0 (m) 141.1141.0 (m), 140.9-140.8 (m), 140.7, 140.6, 140.5, 140.4, 140.3-140.2 (m), 133.9-133.7 (m), 133.4$133.3(\mathrm{~m}), 133.1-133.0(\mathrm{~m}), 129.6-129.4(\mathrm{~m}), 128.1-127.6(\mathrm{~m}), 127.4-127.1(\mathrm{~m}), 77.7,20.9$ ppm. IR: $\tilde{v}$ 3000-2800, 1739, $1692 \mathrm{~cm}^{-1}$. HRMS (ESI) m/z: $[\mathrm{M}+\mathrm{Na}]^{+}$Calcd for $\mathrm{C}_{64} \mathrm{H}_{48} \mathrm{O}_{12} \mathrm{~K}$ 1047.2783; Found 1047.2777. Single crystals of $\mathbf{5 b}$ suitable for X-ray diffraction analysis were grown by diffusion of $\mathrm{MeOH}$ into its THF solution. 


\section{Synthesis of 4,4'-(ethyne-1,2-diyl)dibenzaldehyde 1c}
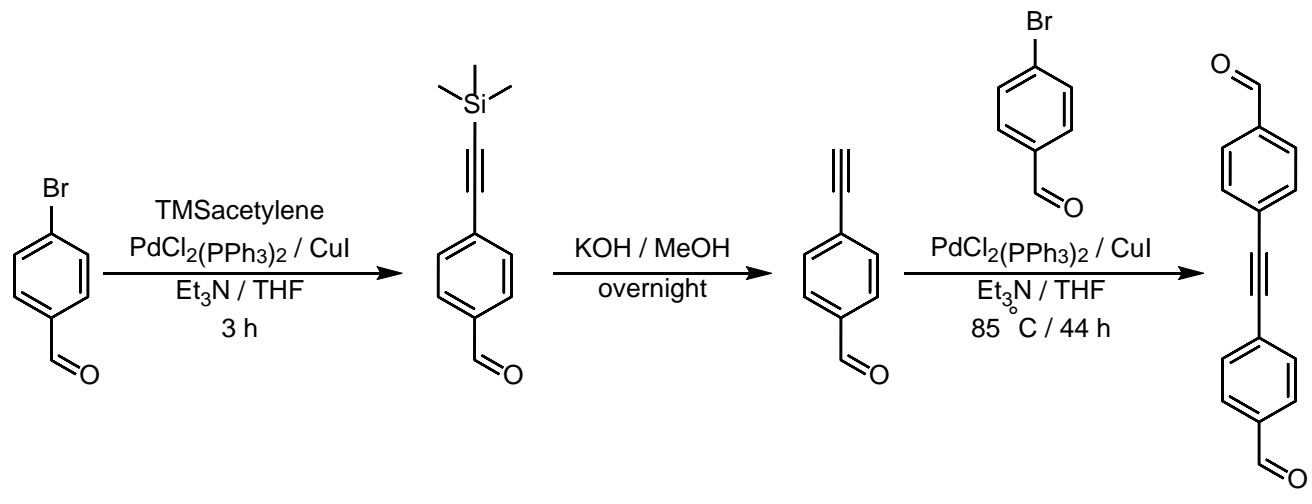

$1 \mathrm{c}$

Synthesis was performed according to a literature procedure, ${ }^{3}$ with some modifications. In a round bottom flask, 4-bromobenzaldehyde (5.00 g, $27.0 \mathrm{mmol}), \mathrm{PdCl}_{2}\left(\mathrm{PPh}_{3}\right)_{2}(379 \mathrm{mg}, 540 \mu \mathrm{mol})$, and CuI (206 mg, $1.08 \mathrm{mmol})$ were dissolved in dry THF $(30 \mathrm{~mL}) . \mathrm{Et}_{3} \mathrm{~N}(4.10 \mathrm{~g}, 5.62 \mathrm{~mL}, 40.5 \mathrm{mmol})$ and trimethylsilylacetylene $(2.79 \mathrm{~g}, 4.04 \mathrm{~mL}, 28.4 \mathrm{mmol})$ were added, and the reaction mixture was stirred for $3 \mathrm{~h}$ at room temperature. The reaction progress was monitored via TLC. After full conversion was achieved, the volatiles were removed under reduced pressure. The obtained crude product was purified by column chromatography on silica gel, eluting with cyclohexane/EtOAc mixture. After evaporation, 4((trimethylsilyl)ethynyl)benzaldehyde (5.42 g, $26.8 \mathrm{mmol}, 99 \%)$ was collected as a dark yellow solid. ${ }^{1} \mathrm{H}$ NMR (400 MHz, CDCl $): \delta 10.01(\mathrm{~s}, 1 \mathrm{H}), 7.82(\mathrm{~d}, J=8.4 \mathrm{~Hz}, 2 \mathrm{H}), 7.61$ (d, J=8.1 Hz, 2H), 0.29-0.27 (m, 9H) ppm. ${ }^{13} \mathrm{C}$ NMR $\left(100 \mathrm{MHz}, \mathrm{CDCl}_{3}\right): \delta 191.4,135.6,132.5,129.4,129.3,103.8,99.0,0.00 \mathrm{ppm}$. IR: $\tilde{v} 2956-2737,2156,1698 \mathrm{~cm}^{-1}$.EIMS $(70 \mathrm{eV}), \mathrm{m} / z(\%): 202(24)[\mathrm{M}+\mathrm{H}]^{+}$.

4-((Trimethylsilyl)ethynyl)benzaldehyde (3.00 g, $14.8 \mathrm{mmol})$ was dissolved in $\mathrm{MeOH}(100 \mathrm{~mL})$, followed by the addition of $\mathrm{KOH}(1.04 \mathrm{~g}, 18.5 \mathrm{mmol})$. The reaction mixture was stirred overnight at room temperature. The reaction progress was monitored via TLC. After full conversion was achieved, brine was added and the product was extracted with EtOAc $(3 \times 100 \mathrm{~mL})$. The combined organic phases were dried over $\mathrm{Na}_{2} \mathrm{SO}_{4}$. All volatiles were removed and the crude product was purified by column chromatography on silica gel, eluting with a cyclohexane/EtOAc mixture. After evaporation, 4ethynylbenzaldehyde (1.92 g, $14.8 \mathrm{mmol}, 89 \%)$ was collected as an orange solid. ${ }^{1} \mathrm{H}$ NMR (400 MHz, $\left.\mathrm{CDCl}_{3}\right): \delta 10.03(\mathrm{~s}, 1 \mathrm{H}), 7.85(\mathrm{~d}, J=8.4 \mathrm{~Hz}, 2 \mathrm{H}), 7.65(\mathrm{~d}, J=8.3 \mathrm{~Hz}, 2 \mathrm{H}), 3.30(\mathrm{~s}, 1 \mathrm{H}) \mathrm{ppm} .{ }^{13} \mathrm{C}$ NMR 
(75 MHz, $\mathrm{CDCl}_{3}$ ): $\delta$ 191.3, 135.9, 132.7 (2C), 129.5 (2C), 128.3, 82.6, 81.0 ppm. IR: $\tilde{v} 3215,2838-$ 2739, 2099, $1682 \mathrm{~cm}^{-1}$. EIMS (70 eV), $m / z(\%): 130(100)[\mathrm{M}+\mathrm{H}]^{+}$.

In a round bottom flask, 4-ethynylbenzaldehyde (2.14 g, $16.4 \mathrm{mmol})$, 4-bromobenzaldehyde (3.04 g, $16.4 \mathrm{mmol}), \mathrm{PdCl}_{2}\left(\mathrm{PPh}_{3}\right)_{2}(346 \mathrm{mg}, 493 \mu \mathrm{mol})$, and CuI $(125 \mathrm{mg}, 657 \mu \mathrm{mol})$ were dissolved in dry THF (119 mL). $\mathrm{Et}_{3} \mathrm{~N}(29.0 \mathrm{~g}, 39.7 \mathrm{~mL}, 287 \mathrm{mmol})$ was added, and the reaction mixture was heated in an $85^{\circ} \mathrm{C}$ oil bath for $44 \mathrm{~h}$. The reaction progress was monitored via TLC. After full conversion was achieved, the reaction mixture was cooled down and the volatiles were removed under reduced pressure. The crude product was purified by column chromatography on silica gel, eluting with $\mathrm{CH}_{2} \mathrm{Cl}_{2}$. After evaporation, 4,4'-(ethyne-1,2-diyl)dibenzaldehyde 1c $(3.06 \mathrm{~g}, 13.1 \mathrm{mmol}, 79 \%)$ was collected as a yellow solid. ${ }^{1} \mathrm{H}$ NMR (400 MHz, $\mathrm{CDCl}_{3}$ ): $\delta 10.05$ (s, 2H), 7.91 (d, J=8.1 Hz, 4H), 7.72 (d, J=8.1 Hz, 4H) ppm. ${ }^{13} \mathrm{C}$ NMR (75 MHz, $\left.\mathrm{CDCl}_{3}\right): \delta 191.3,135.9$, 132.3, 129.6, 128.7, 92.1 ppm. IR: $\tilde{v} 2948-2844$, $1696 \mathrm{~cm}^{-1}$. EIMS (70 eV), $m / z(\%): 234(100)[\mathrm{M}+\mathrm{H}]^{+}$. HRMS (EI) $m / z:[\mathrm{M}]^{+}$Calcd for $\mathrm{C}_{16} \mathrm{H}_{10} \mathrm{O}_{2}$ 234.0675; Found 234.0677. Spectral dara are identical to a previous literature report. ${ }^{3}$ 


\section{Synthesis of Cyclotetrabenzoins $4 c$ and 5c}

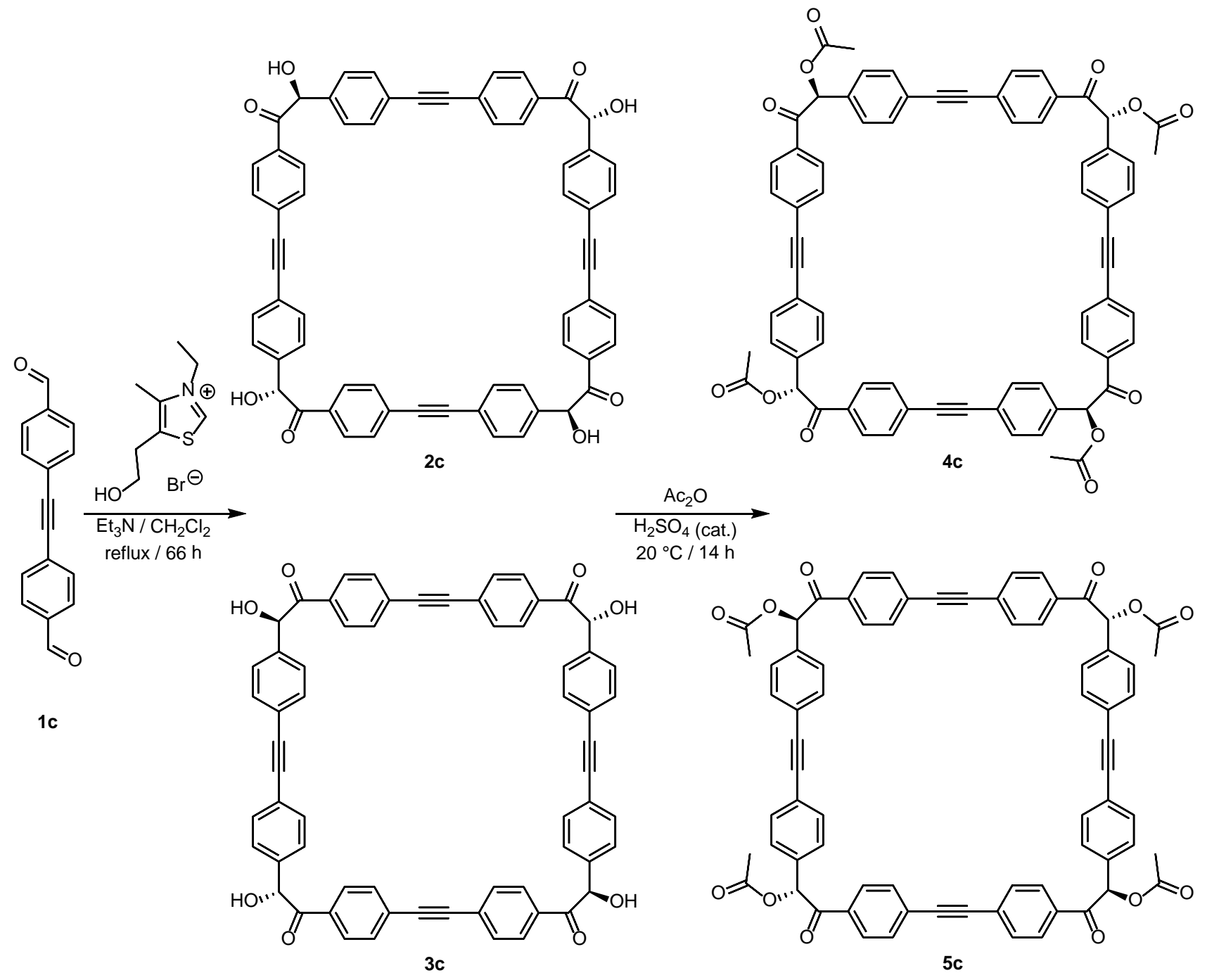

A solution of 4,4'-(ethyne-1,2-diyl)dibenzaldehyde (1 c, $2.00 \mathrm{~g}, 8.54 \mathrm{mmol})$ and 3-ethyl-5-(2hydroxyethyl)-4-methylthiazolium bromide (1.22 g, $4.84 \mathrm{mmol})$ in $\mathrm{CH}_{2} \mathrm{Cl}_{2}(167 \mathrm{~mL})$ was heated to reflux in an oil bath. Then, $\mathrm{Et}_{3} \mathrm{~N}(1.7 \mathrm{~mL})$ was injected and the reaction mixture was heated at reflux for $66 \mathrm{~h}$. While still hot, the solution was filtered, and the residue was washed with $\mathrm{CH}_{2} \mathrm{Cl}_{2}, \mathrm{EtOH}$, and $\mathrm{Et}_{2} \mathrm{O}$. After drying, $1.39 \mathrm{~g}$ of crude products $\mathbf{2 c} / 3 \mathrm{c}$ was obtained as a yellow powder $\left(\mathrm{mp}:>300{ }^{\circ} \mathrm{C}\right.$, decomp.). IR: $\tilde{v} 3452,3424,2215,1677 \mathrm{~cm}^{-1}$.

Crude tolane cyclotetrabenzoins $2 \mathrm{c}$ and $3 \mathrm{c}(1.20 \mathrm{~g}, 1.28 \mathrm{mmol})$ were stirred in $\mathrm{Ac}_{2} \mathrm{O}(75 \mathrm{~mL}, 81.0 \mathrm{~g}$, $793 \mathrm{mmol}$ ) until the mixture turned homogeneous. Catalytic amount of $\mathrm{H}_{2} \mathrm{SO}_{4}$ (6 drops) was added and the mixture was stirred for $18 \mathrm{~h}$ at room temperature. The mixture was poured into $\mathrm{CH}_{2} \mathrm{Cl}_{2}(200 \mathrm{~mL})$, 
transferred to a separatory funnel, washed three times with deionized $\mathrm{H}_{2} \mathrm{O}(150 \mathrm{~mL})$, and twice with brine $(100 \mathrm{~mL})$. The organic layer was dried over anhydrous $\mathrm{Na}_{2} \mathrm{SO}_{4}$, filtered, and the solvent removed via rotary evaporator to provide a yellow, glassy solid. This solid was purified by column chromatography on silica gel, eluting with a $\mathrm{CH}_{2} \mathrm{Cl}_{2} /$ EtOAc mixture. After evaporation, a mixture of compounds 4c and 5c (467 mg, $0.422 \mathrm{mmol}, 23 \%$ overall yield) was collected as a pale yellow solid (mp $>300{ }^{\circ} \mathrm{C}$, decomp.). This mixture could be separated by HPLC (column: reverse phase VDSpher C18 M-SE $5 \mu \mathrm{m}$; $250 \times 4.0 \mathrm{~mm}$; flow rate: $1 \mathrm{~mL} / \mathrm{min}$; injection volume: $5 \mu \mathrm{L}$; detector: $280 \mathrm{~nm}$; solvent gradient: 0-10 min, $\mathrm{MeCN} 80$ to 100\%; 10-21 min, $\mathrm{MeCN} 100 \% ; 21-25 \mathrm{~min}, \mathrm{MeCN} 100$ to 5\%) on an analytical scale, but not preparative. In the absence of X-ray crystal structures, our assignments of structures of $\mathbf{4 c}$ and $\mathbf{5 c}$ are tentative: we cannot tell which compound is which.

Compound 4c: ${ }^{1} \mathrm{H}$ NMR (400 MHz, $\left.\mathrm{CDCl}_{3}\right): \delta 7.86(\mathrm{~m}, 8 \mathrm{H}), 7.47(\mathrm{~m}, 16 \mathrm{H}), 7.40(\mathrm{~m}, 8 \mathrm{H}), 6.82$ (m, 4H), $2.23(\mathrm{t}, J=2.7 \mathrm{~Hz}, 12 \mathrm{H}) \mathrm{ppm} .{ }^{13} \mathrm{C} \mathrm{NMR}\left(100 \mathrm{MHz}, \mathrm{CDCl}_{3}\right): \delta 192.4(\mathrm{~m}), 170.5(\mathrm{~m}), 133.6$ (m), 132.7, 132.6, 132.0, 129.0, 128.9, 128.4, 128.0, 124.3, 124.0, 92.1 (m), 89.8 (m), 21.0 (m) ppm. IR: $\tilde{v}$ 2927-2853, 1742, $1694 \mathrm{~cm}^{-1}$. ESI-MS $(m / z): 1127(100)[\mathrm{M}+\mathrm{Na}]^{+}$. HRMS (ESI) $m / z:[\mathrm{M}+\mathrm{Na}]^{+}$ Calcd for $\mathrm{C}_{72} \mathrm{H}_{48} \mathrm{O}_{12} \mathrm{Na}$ 1127.3043; Found 1127.3092.

Compound 5c: ${ }^{1} \mathrm{H}$ NMR (400 MHz, $\left.\mathrm{CDCl}_{3}\right): \delta 7.86(\mathrm{~m}, 8 \mathrm{H}), 7.48(\mathrm{~m}, 16 \mathrm{H}), 7.40(\mathrm{~m}, 8 \mathrm{H}), 6.82$ (m, 4H), $2.23(\mathrm{t}, J=2.7 \mathrm{~Hz}, 12 \mathrm{H})$ ppm. IR: $\tilde{v} 2927-2853,1742,1694 \mathrm{~cm}^{-1}$. ESI-MS (m/z): 1127 (100) $[\mathrm{M}+\mathrm{Na}]^{+}$. HRMS (ESI) $m / z:[\mathrm{M}+\mathrm{Na}]^{+}$Calcd for $\mathrm{C}_{72} \mathrm{H}_{48} \mathrm{O}_{12} \mathrm{Na}$ 1127.3043; Found 1127.3092. Because of the miniscule amount of pure $\mathbf{5 c}$ that was obtained, a satisfactory ${ }^{13} \mathrm{C}$ NMR spectrum could not be obtained. 


\section{Synthesis of Cyclotetrabenzoins 4d and 5d}

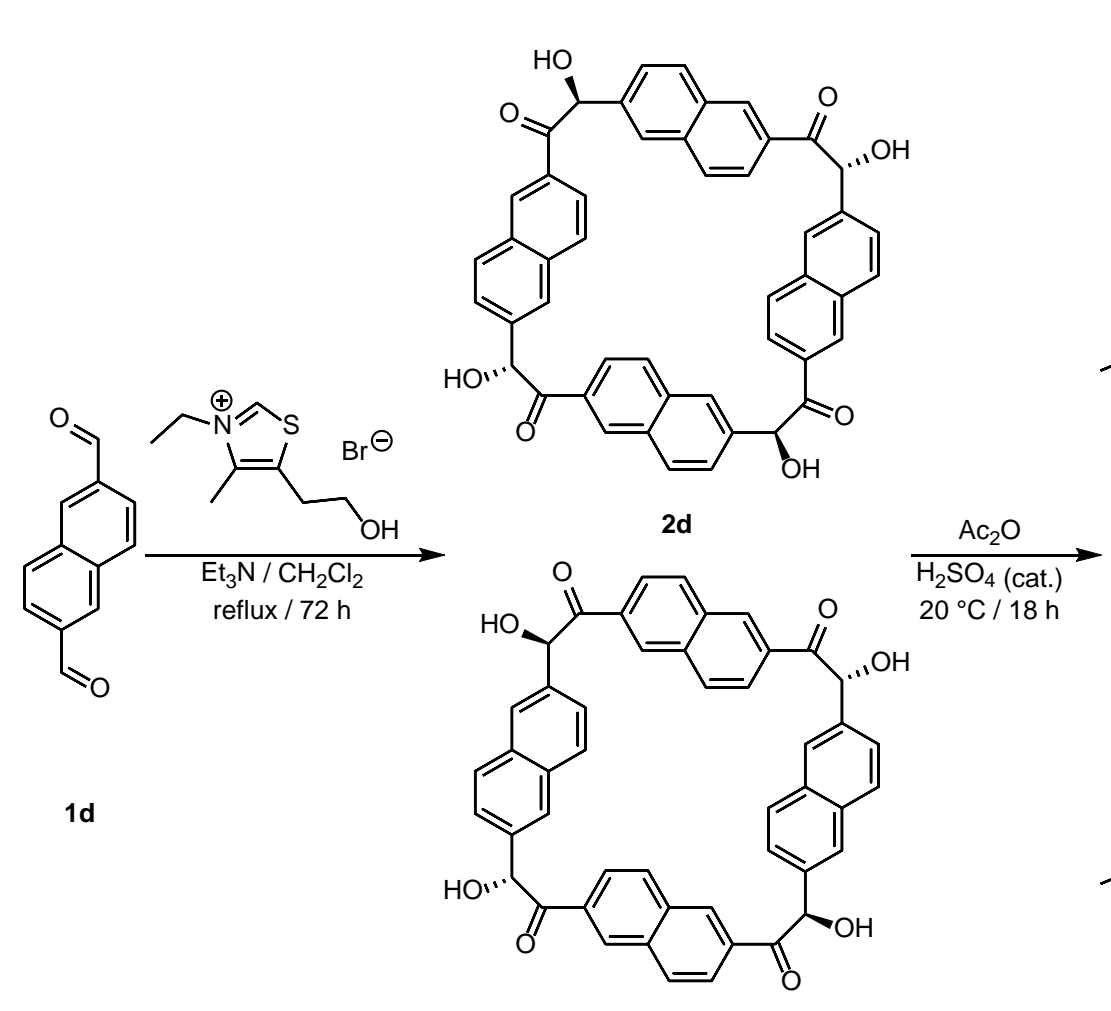

3d
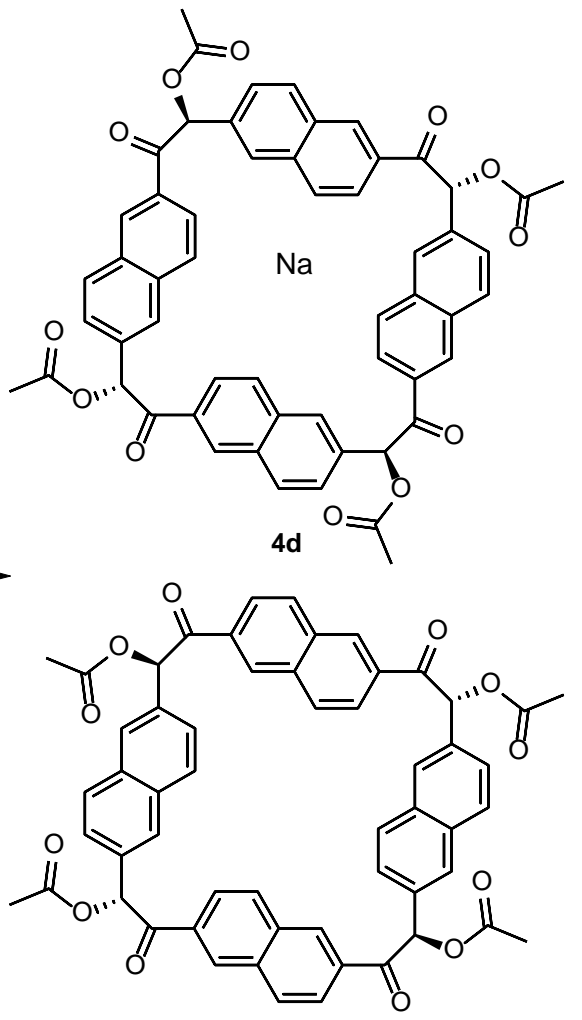

5d

A solution of 2,7-naphthalene dialdehyde (1 d, $1.33 \mathrm{~g}, 7.24 \mathrm{mmol}$ ) and 3-ethyl-5-(2-hydroxyethyl)4-methyl thiazolium bromide $(380 \mathrm{mg}, 1.51 \mathrm{mmol})$ in $\mathrm{CH}_{2} \mathrm{Cl}_{2}(40 \mathrm{~mL})$ was boiled (oil bath) with stirring until all solids dissolved. Then, $\mathrm{Et}_{3} \mathrm{~N}(1.50 \mathrm{~mL}, 1.10 \mathrm{~g}, 10.8 \mathrm{mmol})$ was injected and the solution was kept at reflux for $72 \mathrm{~h}$. While hot, the solution was filtered and washed with $\mathrm{CH}_{2} \mathrm{Cl}_{2}, \mathrm{EtOH}$, and $\mathrm{Et}_{2} \mathrm{O}$. After drying, the collected solids were stirred in a round-bottom flask with $\mathrm{Ac}_{2} \mathrm{O}(3.70 \mathrm{~mL}, 4.00 \mathrm{~g}$, $39.2 \mathrm{mmol}$ ) and a catalytic amount of $\mathrm{H}_{2} \mathrm{SO}_{4}(6 \mathrm{drops})$ at room temperature overnight. The mixture was then neutralized with $1 \mathrm{M}$ solution of $\mathrm{NaOH}(1.50 \mathrm{~m} \mathrm{~mL})$. The resulting solution was then poured into $\mathrm{CH}_{2} \mathrm{Cl}_{2}(100 \mathrm{~mL})$ and washed three times with $\mathrm{H}_{2} \mathrm{O}(25 \mathrm{~mL}$ each $)$ and once with brine $(25 \mathrm{~mL})$. The organic layer was dried over anhydrous $\mathrm{MgSO}_{4}$, filtered, and evaporated with a rotary evaporator. The crude product was purified by column chromatography on silica gel, eluting with 1:1 hexanes/EtOAc mixture. After evaporation, compounds $4 \mathbf{d}\left(R_{\mathrm{f}}=0.27,111 \mathrm{mg}, 122 \mathrm{mmol}\right.$, 9\% overall yield $)$ and $\mathbf{5 d}$ $\left(R_{\mathrm{f}}=0.35,61 \mathrm{mg}, 67.2 \mathrm{mmol}, 5 \%\right.$ overall yield $)$ were collected as white solids $\left(\mathrm{mp}>300{ }^{\circ} \mathrm{C}\right.$, decomp. $)$. 
Single crystals of $\mathbf{4 d}$ suitable for X-ray diffraction grew from one of the column fractions, eluted with EtOAc $/ \mathrm{CH}_{2} \mathrm{Cl}_{2}$, after overnight evaporation.

Compound 4d: ${ }^{1} \mathrm{H}$ NMR (500 MHz, $\left.\mathrm{CDCl}_{3}\right): \delta 8.36-8.22(\mathrm{~m}, 4 \mathrm{H}), 7.99-7.60(\mathrm{~m}, 16 \mathrm{H}), 7.53-7.41$ (m, 4H), 7.14-7.06 (m, 4H), 2.25-2.21 (m, 12H) ppm. ${ }^{13} \mathrm{C}$ NMR (125 MHz, $\left.\mathrm{CDCl}_{3}\right): \delta$ 192.5, 192.3, $192.0,191.8,170.7,170.5,135.5,135.4,135.3,134.5,134.3,133.9,133.8,133.5,133.3,133.0,132.7$, 132.6, 132.4, 132.2, 132.0, 131.9, 131.7, 131.2, 131.0, 130.7, 130.6, 130.3, 130.0, 129.0, 129.5, 129.2, 129.0, 128.9, 128.7, 127.9, 127.0, 126.9, 126.8, 126.5, 126.2, 125.1, 125.0, 124.8, 124.7, 78.0, 77.8, 77.6, 20.9 ppm. HRMS (ESI) $m / z:[\mathrm{M}+\mathrm{Na}]^{+}$Calcd for $\mathrm{C}_{56} \mathrm{H}_{40} \mathrm{O}_{12} \mathrm{Na}$ 927.2417; Found 927.2423.

Compound 5d: ${ }^{1} \mathrm{H}$ NMR (500 MHz, $\left.\mathrm{CDCl}_{3}\right): \delta 8.31(\mathrm{~d}, J=9.7 \mathrm{~Hz}, 1 \mathrm{H}), 8.23(\mathrm{t}, J=8.6 \mathrm{~Hz}, 2 \mathrm{H})$, $8.16(\mathrm{~d}, J=9.7 \mathrm{~Hz}, 1 \mathrm{H}), 7.95-7.86(\mathrm{~m}, 4 \mathrm{H}), 7.82-7.65(\mathrm{~m}, 4 \mathrm{H}), 7.51-7.42(\mathrm{~m}, 12 \mathrm{H}), 7.51-7.42(\mathrm{~m}$, 4H), 7.13-7.07 (m, 4H), $2.23(\mathrm{t}, J=9.2 \mathrm{~Hz}, 12 \mathrm{H})$ ppm. ${ }^{13} \mathrm{C} \mathrm{NMR}\left(125 \mathrm{MHz}, \mathrm{CDCl}_{3}\right): \delta 192.3,192.3$, 192.0, 170.6, 170.5, 135.5, 134.2, 134.1, 134.0, 133.5, 133.4, 133.3, 132.3, 132.0, 131.9, 131.0, 130.4, $130.3,130.2,129.9,129.7,129.6,129.0,128.9,128.9,128.8,126.6,126.5,126.4,125.2,125.0,124.9$, 124.8, 77.8, 77.7, 77.6, 20.9 ppm. HRMS (ESI) $m / z:[\mathrm{M}+\mathrm{Na}]^{+}$Calcd for $\mathrm{C}_{56} \mathrm{H}_{40} \mathrm{O}_{12} \mathrm{Na}$ 927.2417; Found 927.2428. 


\section{Synthesis of Complex 6}
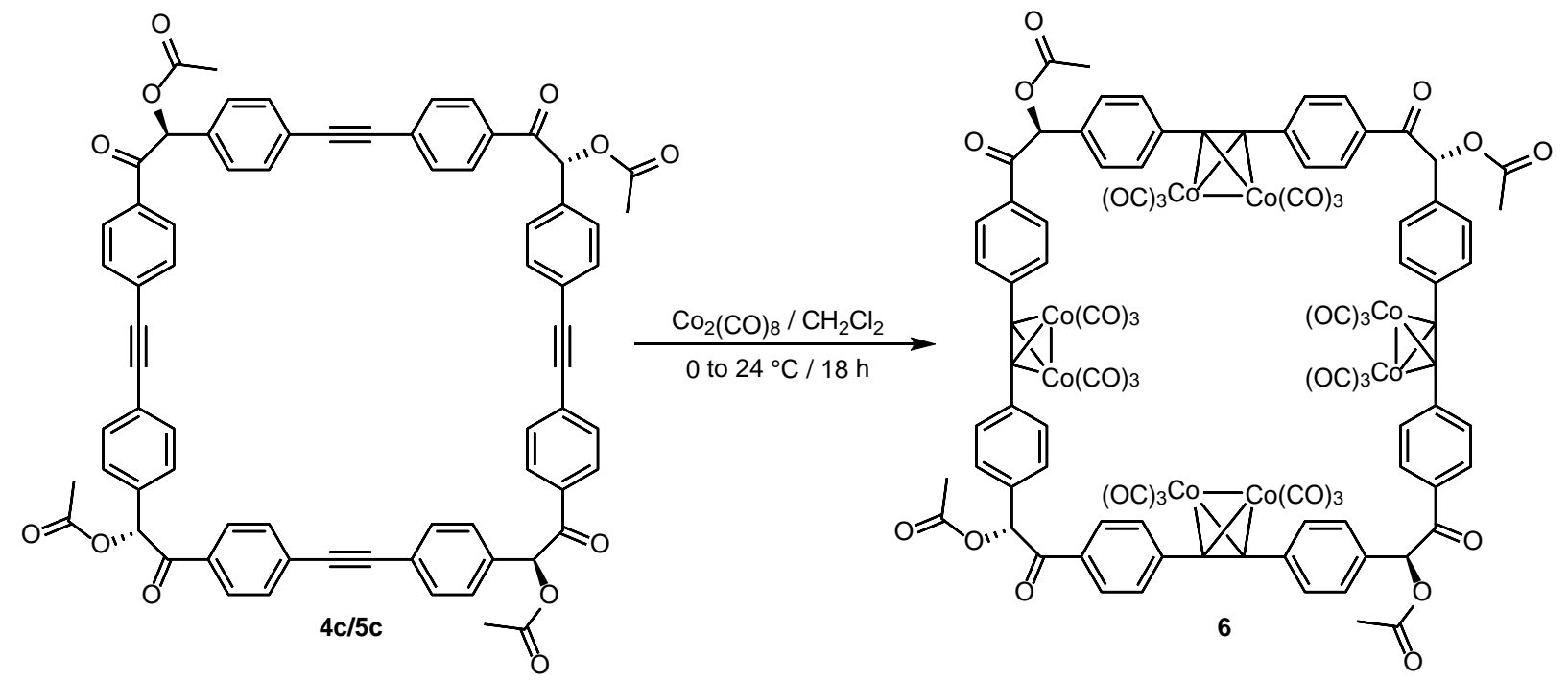

A mixture of acetylated cyclotetrabenzoins $4 \mathbf{c}$ and $\mathbf{5 c}(50.0 \mathrm{mg}, 45.2 \mu \mathrm{mol})$ and $\mathrm{Co}_{2}(\mathrm{CO})_{8}(62.2$ $\mathrm{mg}, 181 \mu \mathrm{mol})$ was dissolved in $\mathrm{CH}_{2} \mathrm{Cl}_{2}(5 \mathrm{~mL})$ at $0{ }^{\circ} \mathrm{C}$. The reaction mixture was stirred at $0{ }^{\circ} \mathrm{C}$ and the reaction progress was monitored via thin-layer chromatography. After $2 \mathrm{~h}$, the reaction mixture was left to warm up to $24{ }^{\circ} \mathrm{C}$ and the stirring was continued overnight. The volatiles were removed via rotary evaporator and the crude product was purified by column chromatography on silica gel, eluting with $\mathrm{CH}_{2} \mathrm{Cl}_{2}$ /EtOAc mixture. After evaporation, compound 6 (55.4 mg, $\left.24.6 \mu \mathrm{mol}, 54 \%\right)$ was collected as a red solid. ${ }^{1} \mathrm{H}$ NMR $\left(500 \mathrm{MHz}, \mathrm{CDCl}_{3}\right): \delta 8.05-7.89(\mathrm{~m}, 8 \mathrm{H}), 7.65-7.41(\mathrm{~m}, 24 \mathrm{H}), 6.86-6.78(\mathrm{~m}, 4 \mathrm{H})$, $2.22(\mathrm{~m}, 12 \mathrm{H})$ ppm. ${ }^{13} \mathrm{C}$ NMR $\left(125 \mathrm{MHz}, \mathrm{CDCl}_{3}\right): \delta 198.6(\mathrm{~m}), 192.7,170.6,144.9(\mathrm{~m}), 139.7(\mathrm{~m})$, $138.2,137.5,133.8,133.2,129.9,129.7,129.4,20.9 \mathrm{ppm}$. Due to the low noise-to-signal ratio, one ${ }^{13} \mathrm{C}$ NMR signal could not be identified despite more than 8,000 collected scans. IR: $\tilde{v} 2933,2092,2053$, 2003, 1742, $1691 \mathrm{~cm}^{-1}$. HRMS (ESI) $\mathrm{m} / z$ : $[\mathrm{M}+\mathrm{I}]^{-}$Calcd for $\mathrm{C}_{96} \mathrm{H}_{48} \mathrm{Co}_{8} \mathrm{O}_{36} \mathrm{I}$ 2375.5660; Found 2375.5651 . 


\section{Synthesis of Compounds $1 \mathrm{f}$ and $1 \mathrm{~h}$}

General procedure for the preparation of the modified reduction agent. ${ }^{4}$ An argon-flushed vial was charged with cis-2,6-dimethylmorpholine $(9.70 \mathrm{~mL}, 78.7 \mathrm{mmol})$ and $\mathrm{PhMe}(84.0 \mathrm{~mL})$. The mixture was stirred and cooled down to $0^{\circ} \mathrm{C}$. The SMEAH solution $(15.0 \mathrm{~mL}, 3.4 \mathrm{~N}$ in PhMe, $52.5 \mathrm{mmol}, 1.00)$ was added dropwise and stirred at $0{ }^{\circ} \mathrm{C}$ for $1 \mathrm{~h}$ to give a colorless homogenous solution.

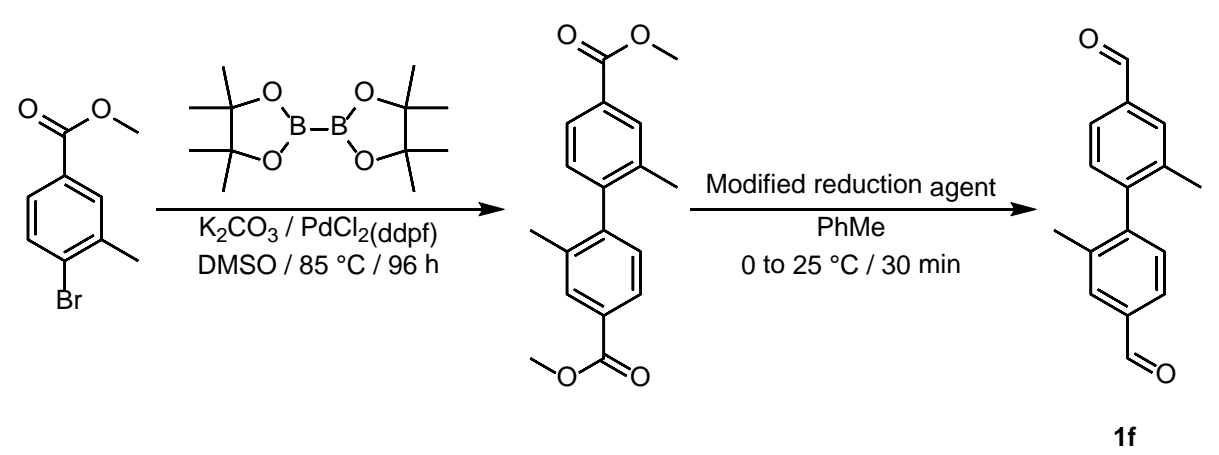

Synthesis of compound $\mathbf{1 f}$ was performed according to a literature procedure. ${ }^{5}$ Bis(pinacolato)diboron (1.09 g, $4.31 \mathrm{mmol})$, 4-bromo-3-methylbenzoic acid methyl ester (2.10 g, 9.81 $\mathrm{mmol}), \mathrm{K}_{2} \mathrm{CO}_{3}(3.78 \mathrm{~g}, 27.4 \mathrm{mmol})$, and $\left[\mathrm{PdCl}_{2}(\mathrm{dppf})\right](0.126 \mathrm{~g}, 0.172 \mathrm{mmol}, 4 \mathrm{~mol} \%)$ were placed into a round bottom flask. Degassed DMSO $(15.4 \mathrm{~mL})$ was added by syringe. The mixture was stirred and heated in an $85^{\circ} \mathrm{C}$ oil bath for $4 \mathrm{~d}$. After cooling, the mixture was poured into $\mathrm{H}_{2} \mathrm{O}(240 \mathrm{~mL})$ and extracted with $\mathrm{CH}_{2} \mathrm{Cl}_{2}(3 \times 67 \mathrm{~mL})$ and EtOAc $(3 \times 100 \mathrm{~mL})$. The combined extracts were washed with $\mathrm{H}_{2} \mathrm{O}(3 \times 80 \mathrm{~mL})$ and brine $(3 \times 80 \mathrm{~mL})$ and dried over anhydrous $\mathrm{Na}_{2} \mathrm{SO}_{4}$. The solution was filtered, and the solvent was removed under reduced pressure. The resulting crude product was purified by column chromatography on silica gel eluting with a cyclohexane/EtOAc mixture. After evaporation, 2,2'dimethyl-1,1'-biphenyl-4,4'-dicarboxylic acid dimethyl ester (1.12 g, $3.76 \mathrm{mmol}, 87 \%)$ was collected as a white solid. ${ }^{1} \mathrm{H}$ NMR (300 MHz, $\mathrm{CDCl}_{3}$ ): $\delta 7.97$ (d, J=1.7 Hz, 2H), 7.90 (dd, J=7.9, $\left.1.7 \mathrm{~Hz}, 2 \mathrm{H}\right) 7.16$ (d, J=7.9 Hz, 2H), 3.94 (s, 6H), 2.08 (s, 6H) ppm. ${ }^{13} \mathrm{C}$ NMR (75 MHz, $\mathrm{CDCl}_{3}$ ): $\delta 167.1,145.4,135.9$, 131.2, 129.5, 129.0, 127.0, 52.1, 19.7 ppm. IR: $\tilde{v} 2952,1710 \mathrm{~cm}^{-1}$. EIMS (70 eV), m/z (\%): 299 (19) $[\mathrm{M}+\mathrm{H}]^{+}, 298(100)[\mathrm{M}]^{+}$.

2,2'-Dimethyl-1,1'-biphenyl-4,4'-dicarboxylic acid dimethyl ester (500 mg, $1.68 \mathrm{mmol}$ ) was dissolved in $\mathrm{PhMe}(17.1 \mathrm{~mL})$ and the solution was cooled to $0{ }^{\circ} \mathrm{C}$. The modified reduction agent $(25.1 \mathrm{~mL}, 12.0$ 
mmol) was added dropwise to prevent a rise in temperature. After addition, the mixture was stirred for $30 \mathrm{~min}$ at room temperature. The reaction was stopped with aqueous $1 \mathrm{~N} \mathrm{HCl}(4.00 \mathrm{~mL})$. The mixture was poured into $\mathrm{H}_{2} \mathrm{O}(50.0 \mathrm{~mL})$ and extracted with EtOAc $(3 \times 30 \mathrm{~mL})$. The combined organic layers were washed with $\mathrm{H}_{2} \mathrm{O}(3 \times 75 \mathrm{~mL})$ and brine $(3 \times 75 \mathrm{~mL})$ and dried over anhydrous $\mathrm{Na}_{2} \mathrm{SO}_{4}$. The solvent was removed under reduced pressure and the crude product was purified by column chromatography on silica gel, eluting with a cyclohexane/EtOAc mixture. After evaporation, compound 1f (348 mg, $1.46 \mathrm{mmol}, 87 \%)$ was collected as a colorless solid. ${ }^{1} \mathrm{H}$ NMR (300 MHz, $\left.\mathrm{CDCl}_{3}\right): \delta 10.04$ (s, 2H), 7.83 (d, J=1.7 Hz, 2H), 7.77 (dd, J=7.7, 1.7 Hz, 2H, ), 7.26 (d, J=7.7 Hz, 2H), 2.12 (s, 6H) ppm. ${ }^{13} \mathrm{C}$ NMR $\left(75 \mathrm{MHz}, \mathrm{CDCl}_{3}\right): \delta 192.1,146.8,136.6,135.9,131.3,129.5,127.4,19.7$ ppm. IR: $\tilde{v} 2815$, 2725, $1688 \mathrm{~cm}^{-1}$. EIMS $(70 \mathrm{eV}), \mathrm{m} / z(\%): 239(19)[\mathrm{M}+\mathrm{H}]^{+}, 238(100)[\mathrm{M}]^{+}$. Spectral data matched a previous literature report. ${ }^{5}$

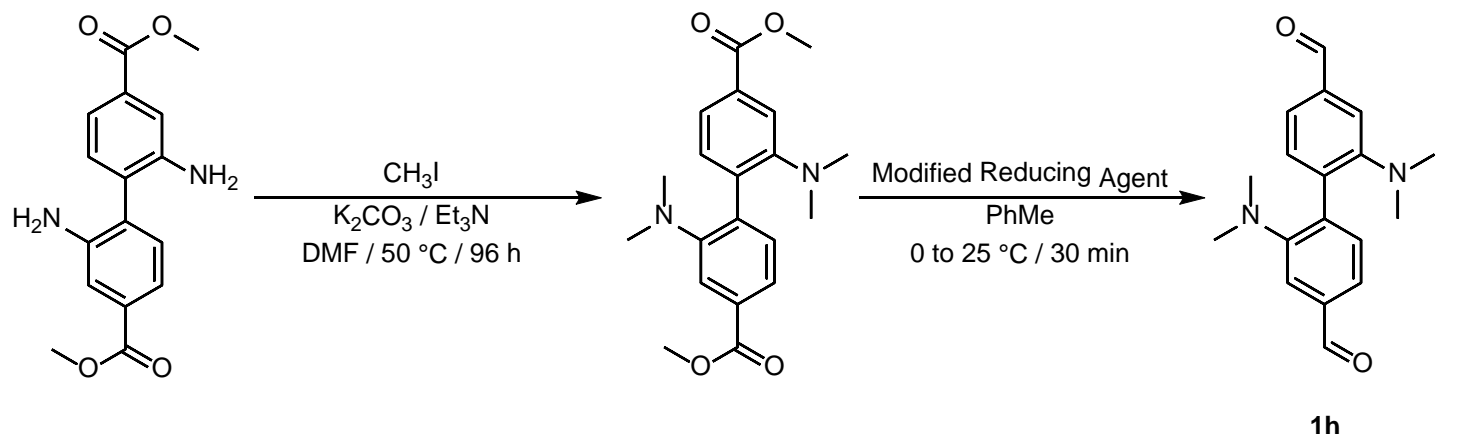

Iodomethane $(0.733 \mathrm{~mL}, 1.67 \mathrm{~g}, 11.8 \mathrm{mmol})$ was added dropwise to a stirred suspension of 2,2'diaminobiphenyl-4,4'-dicarboxylic acid dimethyl ester (0.807 g, $2.69 \mathrm{mmol})$ and $\mathrm{K}_{2} \mathrm{CO}_{3}(2.21 \mathrm{~g}, 16.0$ $\mathrm{mmol})$ in DMF $(10 \mathrm{~mL})$. The mixture was stirred at room temperature and the reaction progress was monitored through thin-layer chromatography. After $18 \mathrm{~h}, 0.5 \mathrm{~mL}$ of $\mathrm{Et}_{3} \mathrm{~N}$ were added, and the mixture was stirred at $50^{\circ} \mathrm{C}$, with an oil bath as a heat source. After $24 \mathrm{~h}, \mathrm{MeI}(0.733 \mathrm{~mL}, 1.67 \mathrm{~g}, 11.8 \mathrm{mmol})$ was added and the mixture was stirred at $50{ }^{\circ} \mathrm{C}$ (oil bath) for $96 \mathrm{~h}$. It was then cooled down and diluted with $\mathrm{H}_{2} \mathrm{O}(10.0 \mathrm{~mL})$ and $\mathrm{NH}_{4} \mathrm{Cl}(10.0 \mathrm{~mL})$. The solution was extracted with EtOAc $(3 \times 20 \mathrm{~mL})$ and the combined organic extracts were washed with $\mathrm{H}_{2} \mathrm{O}(3 \times 10 \mathrm{~mL})$ and brine $(3 \times 10 \mathrm{~mL})$. The organic layers were dried over $\mathrm{Na}_{2} \mathrm{SO}_{4}$ and the solvent was removed under reduced pressure. The resulting crude product was purified by column chromatography on silica gel eluting with a cyclohexane/EtOAc mixture $\left(+0.1 \% \mathrm{Et}_{3} \mathrm{~N}\right)$. After evaporation, 2,2'-dimethyldiamino-1,1'-biphenyl-4,4'-dicarboxylic acid 
dimethyl ester (670 mg, $1.88 \mathrm{mmol}, 79 \%)$ was collected as a yellow oil. ${ }^{1} \mathrm{H}$ NMR $\left(300 \mathrm{MHz}, \mathrm{CDCl}_{3}\right): \delta$ 7.68 (d, J=1.7 Hz, 2H), 7.59 (dd, J=8.1, $1.5 \mathrm{~Hz}, 2 \mathrm{H}$ ), 7.44 (d, J=8.0 Hz, 2H), 3.91 (s, 6H), 2.59 (s, 12H) ppm. ${ }^{13} \mathrm{C}$ NMR $\left(75 \mathrm{MHz}, \mathrm{CDCl}_{3}\right): \delta 167.3,150.2,136.6,131.1,129.7,121.8,119.2,52.1,42.7 \mathrm{ppm}$. IR: $\tilde{v} 2942,2833,2783,1715 \mathrm{~cm}^{-1}$. EIMS (70 eV), $m / z(\%): 356(0.28)[\mathrm{M}]^{+}$.

2,2'-Dimethyldiaminobiphenyl-4,4'-dicarboxylic acid dimethyl ester (540 mg, $1.52 \mathrm{mmol}$ ) was dissolved in $\mathrm{PhMe}(15.0 \mathrm{~mL})$ and cooled to $0{ }^{\circ} \mathrm{C}$. The modified reduction agent $(22.8 \mathrm{~mL}, 10.9 \mathrm{mmol})$ was added dropwise to prevent a rise in temperature. After addition, the mixture was stirred for $30 \mathrm{~min}$ at room temperature. The reaction was stopped with aqueous $1 \mathrm{~N} \mathrm{HCl}(3.70 \mathrm{~mL})$. The mixture was poured into $\mathrm{H}_{2} \mathrm{O}(50 \mathrm{~mL})$ and extracted with EtOAc $(3 \times 30 \mathrm{~mL})$. The combined organic layers were washed with $\mathrm{H}_{2} \mathrm{O}(3 \times 75 \mathrm{~mL})$ and brine $(3 \times 75 \mathrm{~mL})$ and dried over anhydrous $\mathrm{Na}_{2} \mathrm{SO}_{4}$. The solvent was removed under reduced pressure and the crude product was purified by column chromatography on silica gel eluting with cyclohexane/EtOAc mixture. After evaporation, compound $\mathbf{1 h}$ (370 $\mathrm{mg}, 1.23$ mmol, $86 \%$ ) was collected as a yellow solid. ${ }^{1} \mathrm{H}$ NMR (300 MHz, $\left.\mathrm{CDCl}_{3}\right): \delta 9.99(\mathrm{~s}, 2 \mathrm{H}), 7.57(\mathrm{~d}, J=7.8$ $\mathrm{Hz}, 2 \mathrm{H}), 7.53$ (d, J=1.6 Hz, 2H), 7.44 (dd, J=7.8, $1.6 \mathrm{~Hz}, 2 \mathrm{H}), 2.64(\mathrm{~s}, 12 \mathrm{H}) \mathrm{ppm} .{ }^{13} \mathrm{C} \mathrm{NMR}(75 \mathrm{MHz}$, $\left.\mathrm{CDCl}_{3}\right): \delta 192.3,150.7,137.8,136.4,131.6,122.9,117.9,42.7$ ppm. IR: $\tilde{v} 2841,2784,1691 \mathrm{~cm}^{-1}$. EIMS $(70 \mathrm{eV}), m / z(\%): 296(27)[\mathrm{M}]^{+}$. Spectral data matches a previous literature report. ${ }^{6}$

\section{Synthesis of Compound $1 \mathrm{~g}$}

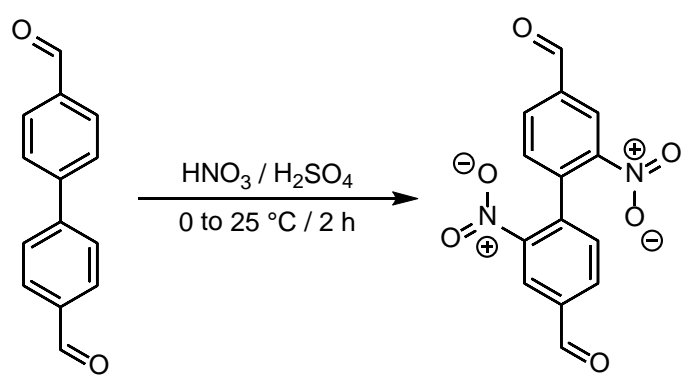

19

The synthesis was performed according to a literature procedure. ${ }^{6}$ Nitric $(1.29 \mathrm{~mL}, 1.80 \mathrm{~g}, 28.6$ $\mathrm{mmol}$ ) and sulfuric $(1.67 \mathrm{~mL}, 3.02 \mathrm{~g}, 30.8 \mathrm{mmol})$ acids were mixed, cooled to $-5{ }^{\circ} \mathrm{C}$, and stirred vigorously. 1,1'-Biphenyl-4,4'-dialdehyde (363 mg, $1.73 \mathrm{mmol}$ ) was slowly added to prevent the temperature from exceeding $0{ }^{\circ} \mathrm{C}$. After addition, the mixture was stirred for $1 \mathrm{~h}$ at $0{ }^{\circ} \mathrm{C}$ and $1 \mathrm{~h}$ at room 
temperature. The mixture was then poured into iced $\mathrm{H}_{2} \mathrm{O}(46 \mathrm{~mL})$, and the precipitated solid was obtained through filtration and dissolved in $\mathrm{CHCl}_{3}(50 \mathrm{~mL})$. This solution was dried over anhydrous $\mathrm{Na}_{2} \mathrm{SO}_{4}$. The solution was heated at reflux with silica gel $(300 \mathrm{mg})$ and filtered hot twice. The solvent was removed under reduced pressure and the crude product was purified by column chromatography on silica gel, eluting with a cyclohexane/EtOAc mixture. After evaporation, compound $\mathbf{1 g}$ (357 mg, 1.19 mmol, $69 \%)$ was collected as a colorless solid. ${ }^{1} \mathrm{H} \operatorname{NMR}\left(300 \mathrm{MHz}, \mathrm{CDCl}_{3}\right): \delta 10.16(\mathrm{~s}, 2 \mathrm{H}), 8.75(\mathrm{~d}$, $J=1.6 \mathrm{~Hz}, 2 \mathrm{H}), 8.25(\mathrm{dd}, J=7.8,1.6 \mathrm{~Hz}, 2 \mathrm{H}), 7.51(\mathrm{~d}, J=7.8 \mathrm{~Hz}, 2 \mathrm{H}) \mathrm{ppm} .{ }^{13} \mathrm{C} \mathrm{NMR}\left(75 \mathrm{MHz}, \mathrm{CDCl}_{3}\right) \delta$ 189.1, 147.3, 138.6, 137.2, 133.5, 131.5, 126.0 ppm. IR: $\tilde{v} 3079,2949,1699 \mathrm{~cm}^{-1}$. EIMS (70 eV), m/z (\%): $300(6)[\mathrm{M}]^{+}, 254(100)\left[\mathrm{C}_{14} \mathrm{H}_{8} \mathrm{NO}_{4}\right]^{+}$. Spectral data matches a previous literature report. ${ }^{6}$

\section{Synthesis of Compound 1i}

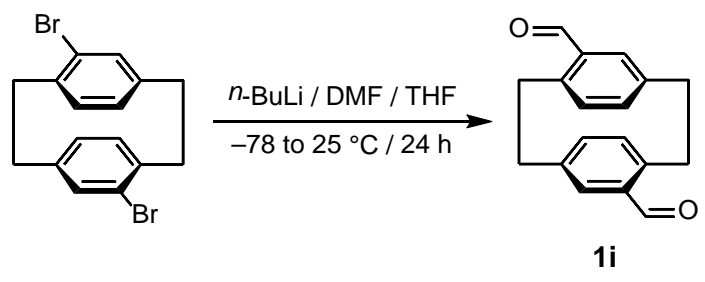

Synthesis followed a published literature procedure. ${ }^{7}$ 4,16-Dibromo[2.2] paracyclophane (0.864 g, $2.36 \mathrm{mmol})$ was dissolved in degassed THF $(22.5 \mathrm{~mL})$. The solution was stirred and cooled to $-78^{\circ} \mathrm{C}$ and then $n$-BuLi solution (3.14 mL, $2.5 \mathrm{M}, 0.503 \mathrm{~g}, 7.86 \mathrm{mmol}$ ) was added dropwise. The mixture was stirred at $-78^{\circ} \mathrm{C}$ for $2 \mathrm{~h}$, then DMF $(2.72 \mathrm{~mL}, 2.59 \mathrm{~g}, 35.4 \mathrm{mmol})$ was added dropwise and the mixture was left to warm up room temperature overnight. The solution was washed with brine $(3 \times 7.5 \mathrm{~mL})$ and $\mathrm{NaHCO}_{3}(3 \times 7.5 \mathrm{~mL})$, dried over $\mathrm{Na}_{2} \mathrm{SO}_{4}$, and the solvent was removed under reduced pressure. The crude product was purified by column chromatography on silica gel, eluting with an $n$-hexane $/ \mathrm{CH}_{2} \mathrm{Cl}_{2}$ mixture. After evaporation, compound $\mathbf{1 i}(195 \mathrm{mg}, 0.737 \mathrm{mmol}, 31 \%)$ was collected as a colorless solid. ${ }^{1} \mathrm{H}$ NMR (300 MHz, CDCl $): \delta 9.93(\mathrm{~s}, 2 \mathrm{H}), 7.05$ (d, J=2.0 Hz, 2H), 6.63 (dd, J=7.8, 2.0 Hz, 2H), 6.52 (d, $J=7.8 \mathrm{~Hz}, 2 \mathrm{H}$ ), 4.13 (ddd, $J=12.9,10.2,2.4 \mathrm{~Hz}, 2 \mathrm{H}$ ), 3.29 (ddd, $J=13.1,10.6,2.4 \mathrm{~Hz}, 2 \mathrm{H}$ ), 3.15 (ddd, $J=13.1,10.1,5.8 \mathrm{~Hz}, 2 \mathrm{H}), 3.01$ (ddd, $J=13.1,10.6,5.8 \mathrm{~Hz}, 2 \mathrm{H})$ ppm. ${ }^{13} \mathrm{C}$ NMR $\left(75 \mathrm{MHz}, \mathrm{CDCl}_{3}\right): \delta$ 191.9, 142.9, 140.5, 137.0, 136.8, 136.6, 135.2, 34.4, 32.8 ppm. IR: $\tilde{v} 2930-2747,1668 \mathrm{~cm}^{-1}$. EIMS (70 $\mathrm{eV}), m / z(\%): 264(17)[\mathrm{M}]^{+}$. Spectral data match a previous literature report. ${ }^{8}$ 


\section{Crystal Data and Structure Refinement Parameters for Compound 5b}

Empirical formula

Formula weight

Temperature

Wavelength

Crystal system

Space group

Unit cell dimensions

Volume

Z

Density (calculated)

Absorption coefficient

$F(000)$

Crystal size

Theta range for data collection

Index ranges

Reflections collected

Independent reflections

Completeness to theta $=66.694^{\circ}$

Absorption correction

Max. and min. transmission

Refinement method

Data / restraints / parameters

Goodness-of-fit on $F^{2}$

Final $R$ indices $[I>2 \sigma(I)]$

$R$ indices (all data)

Absolute structure parameter

Largest diff. peak and hole
$\mathrm{C}_{64} \mathrm{H}_{48} \mathrm{O}_{12}$

$1009.02 \mathrm{~g} \mathrm{~mol}^{-1}$

$123(2) \mathrm{K}$

$1.54178 \AA$

Orthorhombic

Fdd2

$a=13.3250(4) \AA \quad a=90^{\circ}$

$b=25.7868(7) \AA \quad \beta=90^{\circ}$

$c=51.1388(15) \AA \quad \gamma=90^{\circ}$

17571.8(9) $\AA^{3}$

8

$0.763 \mathrm{Mg} \mathrm{m}^{-3}$

$0.429 \mathrm{~mm}^{-1}$

4224

$0.16 \times 0.14 \times 0.01 \mathrm{~mm}^{3}$

3.833 to $66.694^{\circ}$

$-11 \leq h \leq 15,-30 \leq k \leq 20,-52 \leq l \leq 58$

16314

$6701[R($ int $)=0.0288]$

$98.3 \%$

Empirical

0.7528 and 0.5708

Full-matrix least-squares on $F^{2}$

$6701 / 310 / 364$

1.224

$R_{1}=0.0640, w R_{2}=0.1670$

$R_{1}=0.1215, \mathrm{w} R_{2}=0.1934$

$0.7(5)$

0.133 and $-0.128 \mathrm{e}^{-} \AA^{-3}$ 
Figure S2. Thermal ellipsoid plot of the X-ray crystal structure of compound $\mathbf{5 b}$. Ellipsoids shown at $50 \%$ probability.

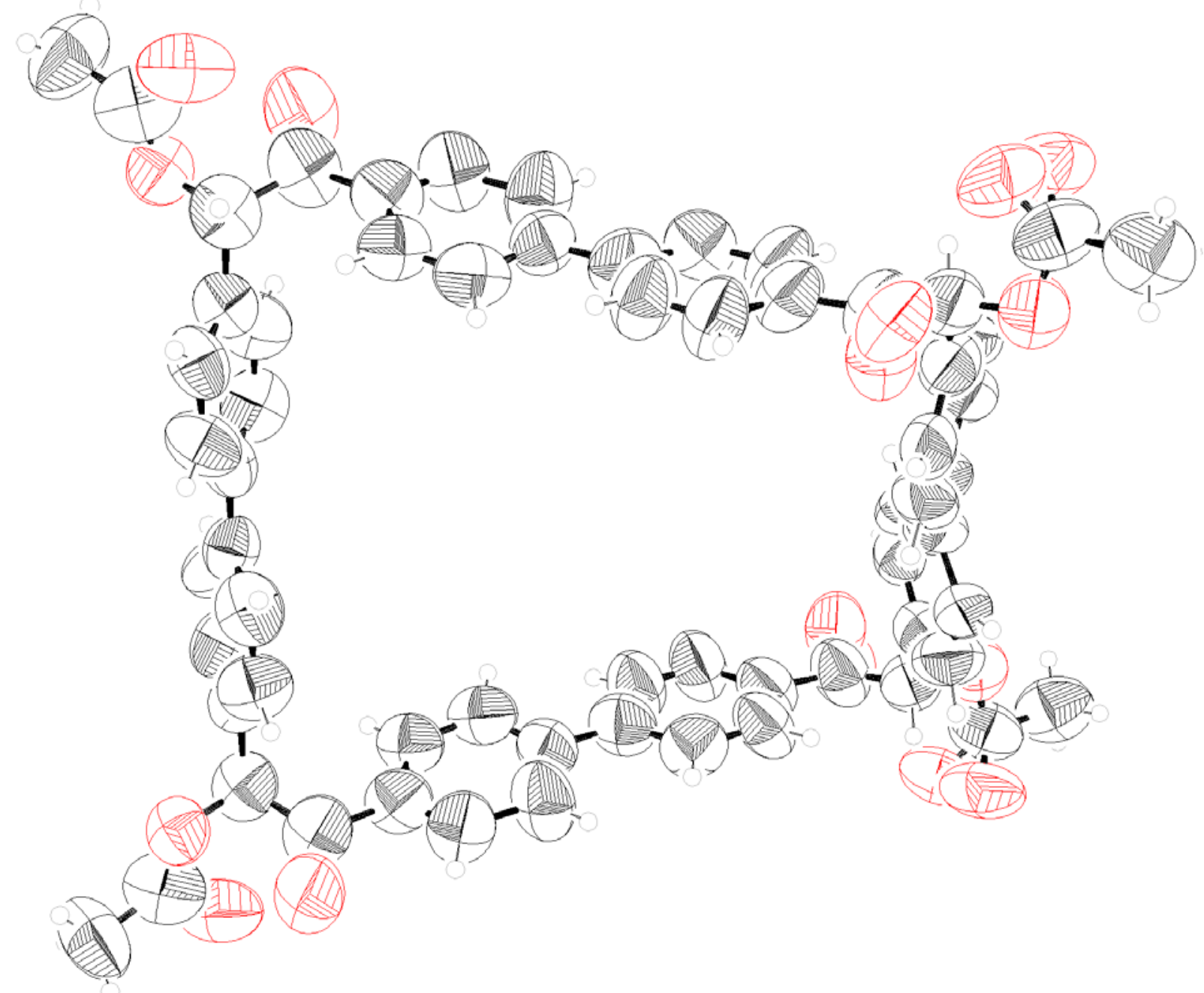




\section{Crystal Data and Structure Refinement Parameters for Compound 4d}

\begin{tabular}{|c|c|c|}
\hline Empirical formula & \multicolumn{2}{|l|}{$\mathrm{C}_{56} \mathrm{H}_{40} \mathrm{O}_{12}$} \\
\hline Formula weight & \multicolumn{2}{|l|}{$904.88 \mathrm{~g} \mathrm{~mol}^{-1}$} \\
\hline Temperature & \multicolumn{2}{|l|}{$123(2) \mathrm{K}$} \\
\hline Wavelength & \multicolumn{2}{|l|}{$1.54178 \AA$} \\
\hline Crystal system & \multicolumn{2}{|l|}{ Tetragonal } \\
\hline Space group & \multicolumn{2}{|l|}{$I \overline{4}$} \\
\hline \multirow[t]{3}{*}{ Unit cell dimensions } & $a=16.0194(7) \AA$ & $a=90^{\circ}$ \\
\hline & $b=16.0194(7) \AA$ & $\beta=90^{\circ}$ \\
\hline & $c=10.8949(5) \AA$ & $\gamma=90^{\circ}$ \\
\hline Volume & \multicolumn{2}{|l|}{$2795.9(3) \AA^{3}$} \\
\hline$Z$ & \multicolumn{2}{|l|}{2} \\
\hline Density (calculated) & \multicolumn{2}{|l|}{$1.075 \mathrm{Mg} \mathrm{m}^{-3}$} \\
\hline Absorption coefficient & \multicolumn{2}{|l|}{$0.623 \mathrm{~mm}^{-1}$} \\
\hline$F(000)$ & \multicolumn{2}{|l|}{944} \\
\hline Crystal size & \multicolumn{2}{|c|}{$0.09 \times 0.07 \times 0.06 \mathrm{~mm}^{3}$} \\
\hline Theta range for data collection & \multicolumn{2}{|l|}{3.902 to $68.262^{\circ}$} \\
\hline Index ranges & \multicolumn{2}{|c|}{$-19 \leq h \leq 18,-19 \leq k \leq 19,-13 \leq l \leq 12$} \\
\hline Reflections collected & \multicolumn{2}{|l|}{9810} \\
\hline Independent reflections & \multicolumn{2}{|c|}{$2555[R($ int $)=0.0273]$} \\
\hline Completeness to theta $=67.679^{\circ}$ & \multicolumn{2}{|l|}{$99.9 \%$} \\
\hline Absorption correction & \multicolumn{2}{|l|}{ Empirical } \\
\hline Max. and min. transmission & \multicolumn{2}{|l|}{0.7531 and 0.6447} \\
\hline Refinement method & \multicolumn{2}{|c|}{ Full-matrix least-squares on $F^{2}$} \\
\hline Data / restraints / parameters & \multicolumn{2}{|l|}{$2555 / 0 / 156$} \\
\hline Goodness-of-fit on $F^{2}$ & \multicolumn{2}{|l|}{1.068} \\
\hline Final $R$ indices $[I>2 \sigma(I)]$ & \multicolumn{2}{|c|}{$R_{1}=0.0405, \mathrm{w} R_{2}=0.1115$} \\
\hline$R$ indices (all data) & \multicolumn{2}{|c|}{$R_{1}=0.0432, \mathrm{w} R_{2}=0.1138$} \\
\hline Absolute structure parameter & \multicolumn{2}{|l|}{$0.2(3)$} \\
\hline Largest diff. peak and hole & \multicolumn{2}{|c|}{0.149 and $-0.162 \mathrm{e}^{-} \AA^{-3}$} \\
\hline
\end{tabular}


Figure S3. Thermal ellipsoid plot of the X-ray crystal structure of compound 4d. Ellipsoids shown at $50 \%$ probability.

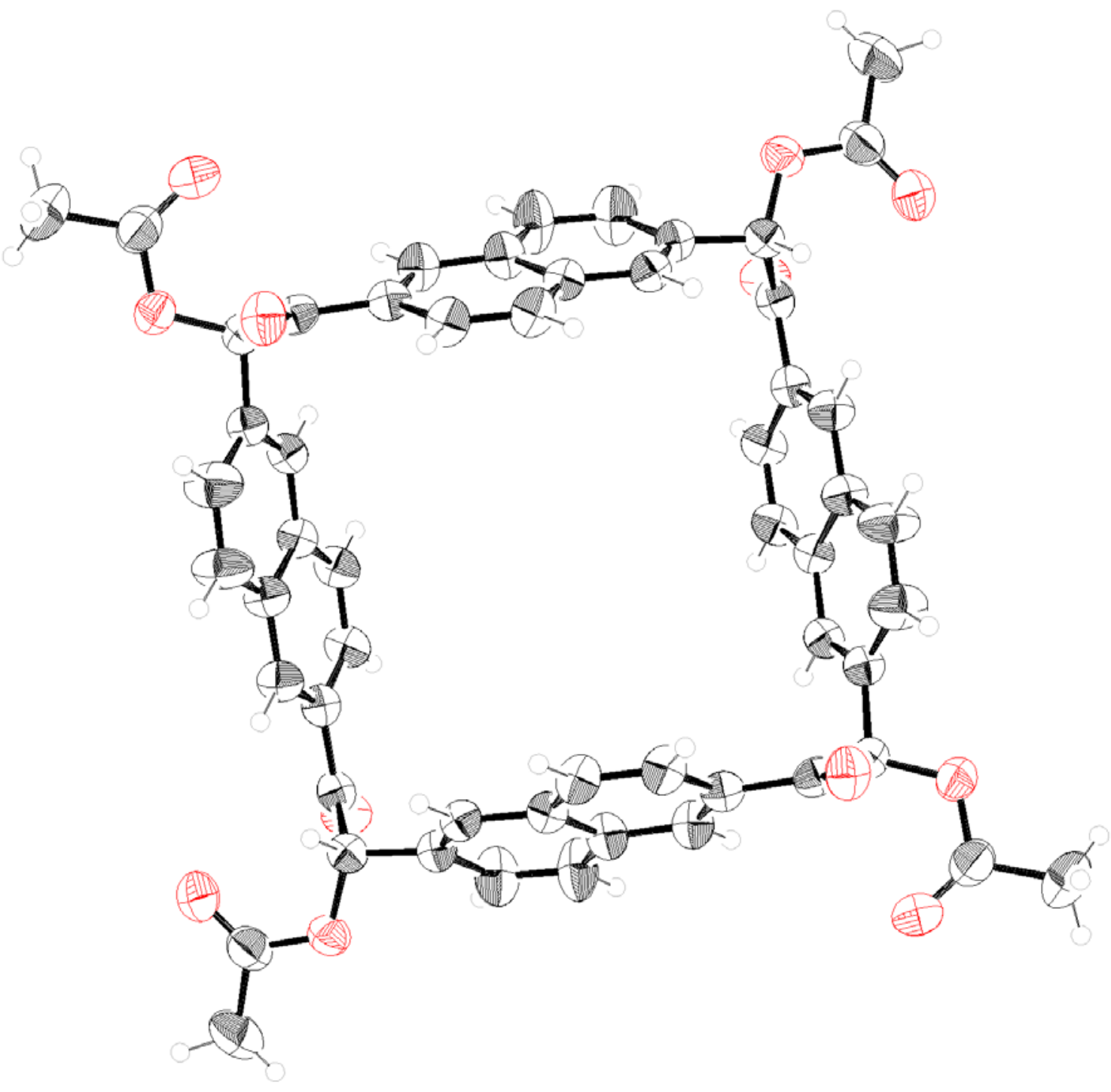




\section{${ }^{1} \mathrm{H}$ and ${ }^{13} \mathrm{C}$ NMR Spectra of Cyclotetrabenzoin $5 b$}

Figure S4. ${ }^{1} \mathrm{H}$ NMR Spectrum of compound $\mathbf{5 b}\left(600 \mathrm{MHz}, \mathrm{CDCl}_{3}, 25^{\circ} \mathrm{C}\right)$.

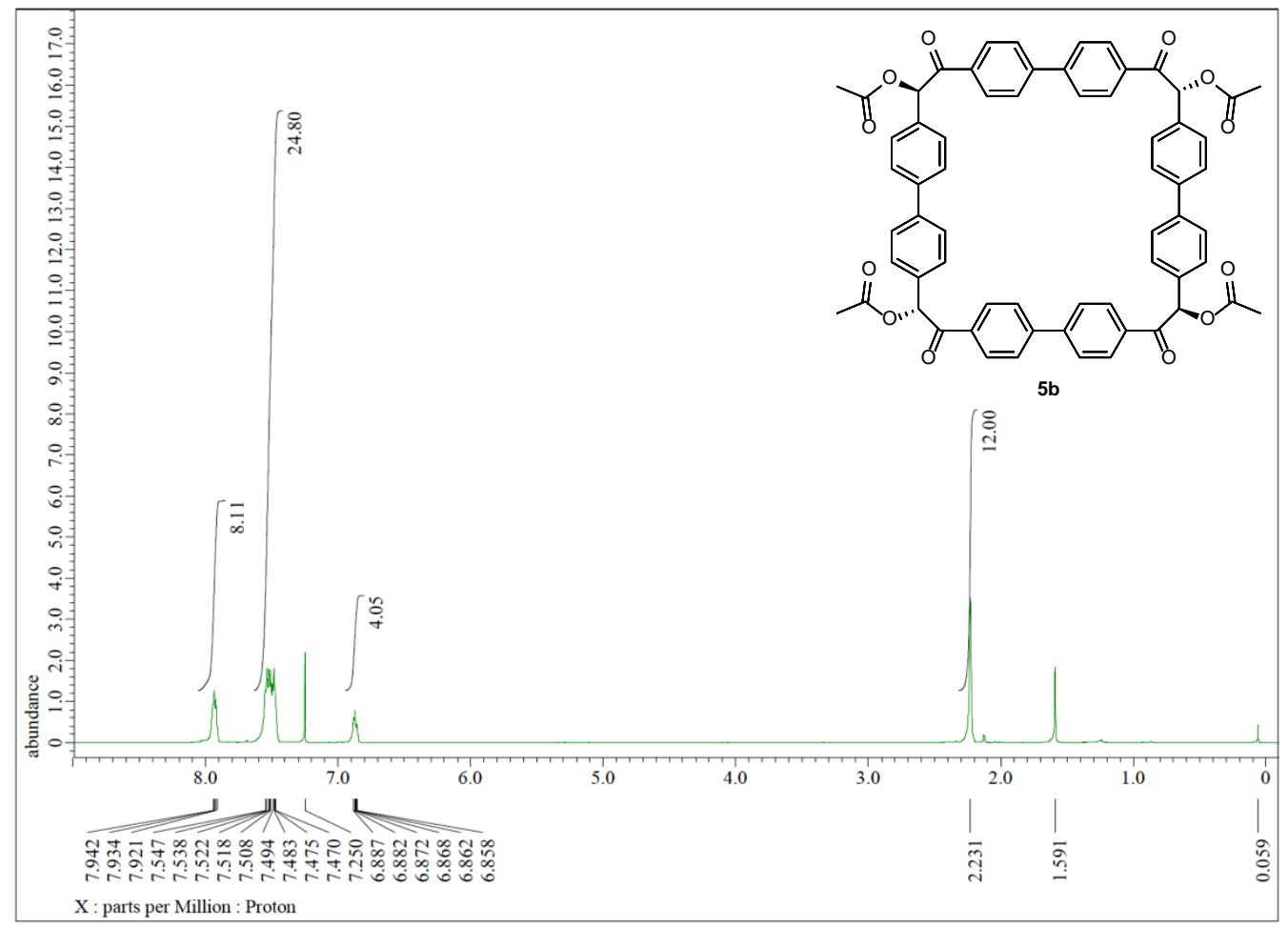

Figure S5. ${ }^{13} \mathrm{C}$ NMR Spectrum of compound $\mathbf{5 b}\left(150 \mathrm{MHz}, \mathrm{CDCl}_{3}, 25^{\circ} \mathrm{C}\right)$.

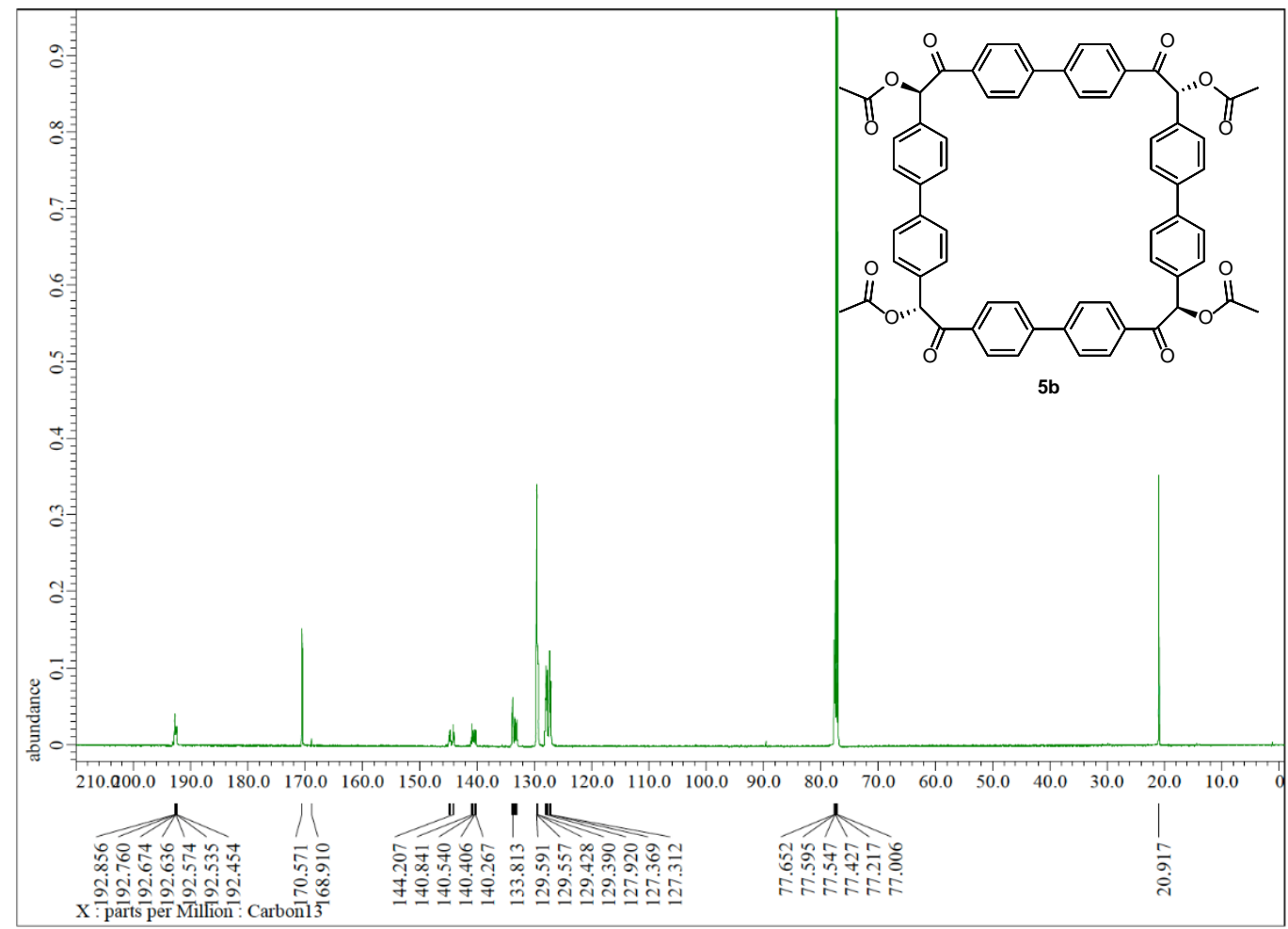




\section{${ }^{1} \mathrm{H}$ and ${ }^{13} \mathrm{C}$ NMR Spectra of Cyclotetrabenzoins $4 \mathrm{c}$ and 5c}

Figure S6. ${ }^{1} \mathrm{H} \mathrm{NMR}$ Spectrum of compound $4 \mathbf{c}\left(400 \mathrm{MHz}, \mathrm{CDCl}_{3}, 25^{\circ} \mathrm{C}\right)$.

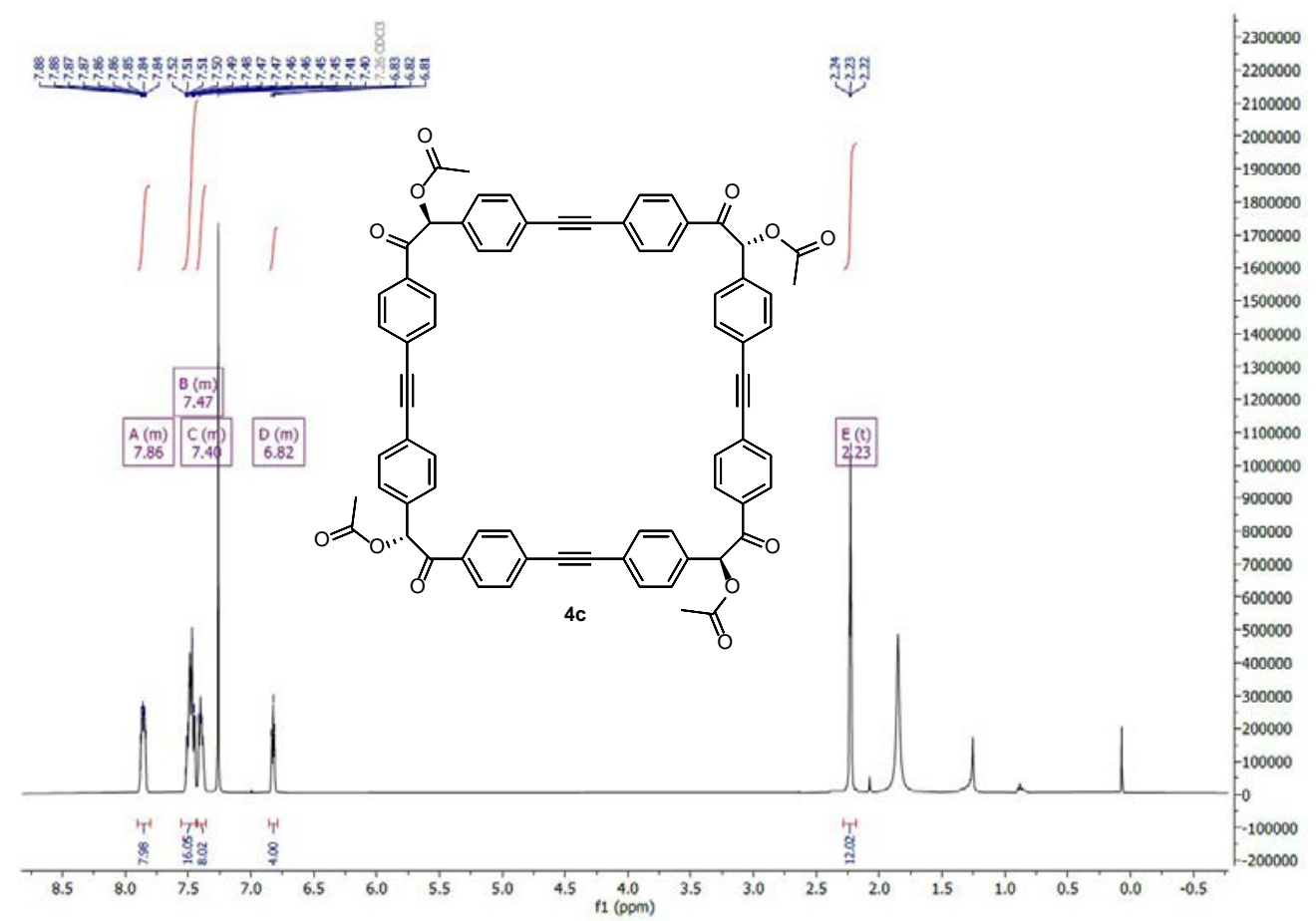

Figure S7. ${ }^{13} \mathrm{C}$ NMR Spectrum of compound $4 \mathbf{c}\left(100 \mathrm{MHz}, \mathrm{CDCl}_{3}, 25^{\circ} \mathrm{C}\right)$.

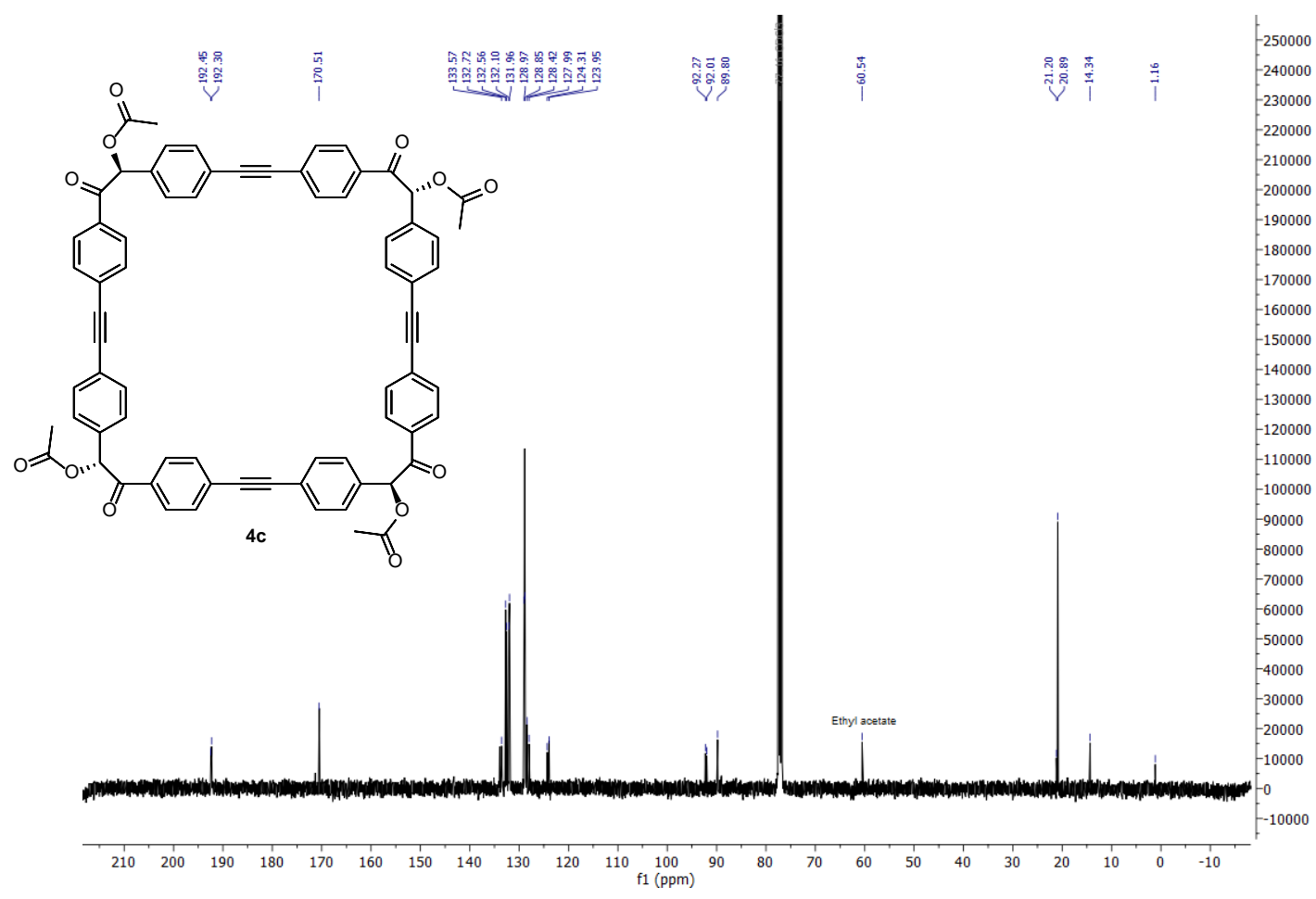


Figure S8. ${ }^{1} \mathrm{H}$ NMR Spectrum of compound $5 \mathbf{c}\left(400 \mathrm{MHz}, \mathrm{CDCl}_{3}, 25^{\circ} \mathrm{C}\right)$.

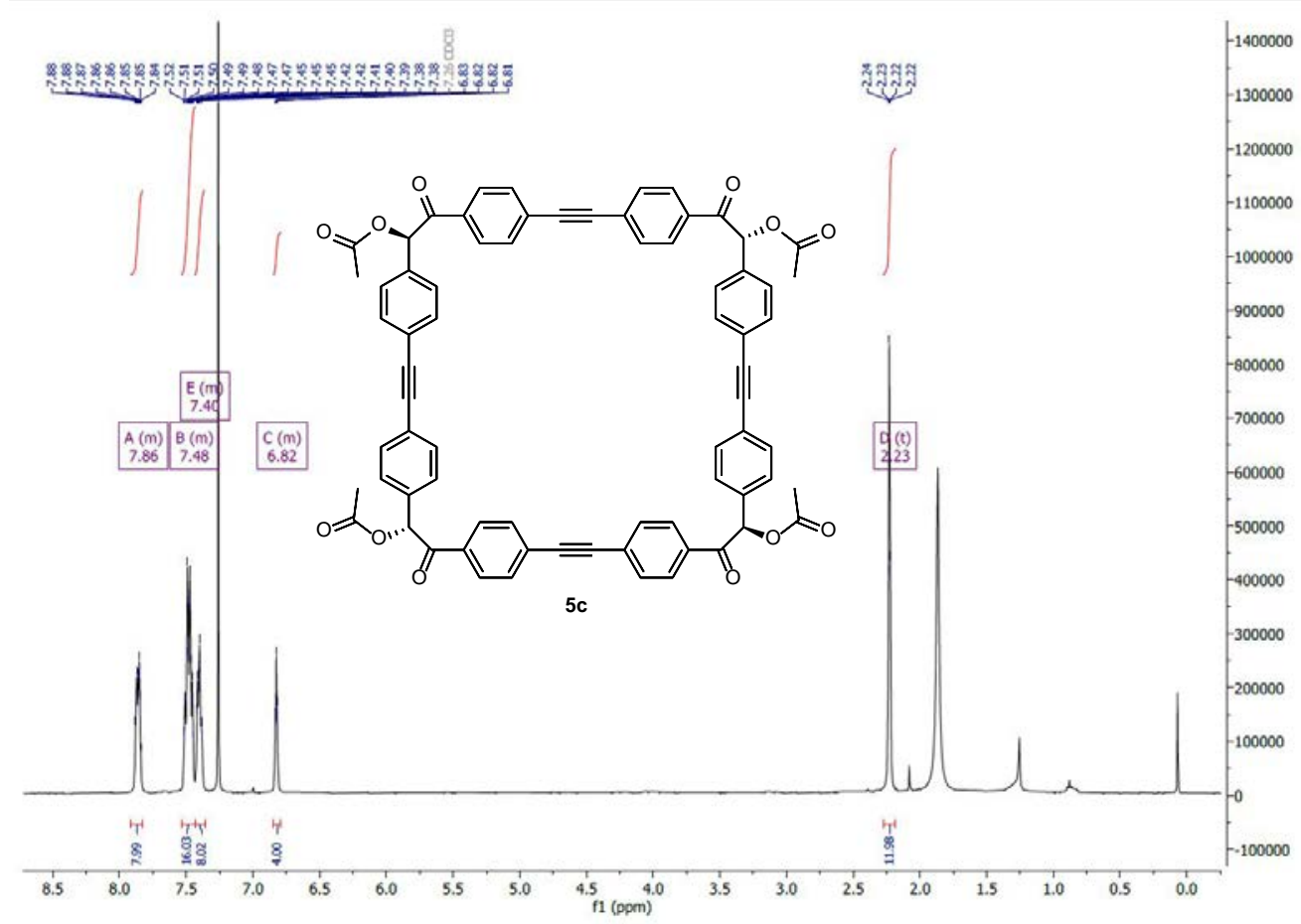




\section{${ }^{1} \mathrm{H}$ and ${ }^{13} \mathrm{C}$ NMR Spectra of Cyclotetrabenzoin 4d and 5d}

Figure S10. ${ }^{1} \mathrm{H}$ Spectrum of compound $4 \mathbf{d}\left(500 \mathrm{MHz}, \mathrm{CDCl}_{3}, 25^{\circ} \mathrm{C}\right)$.

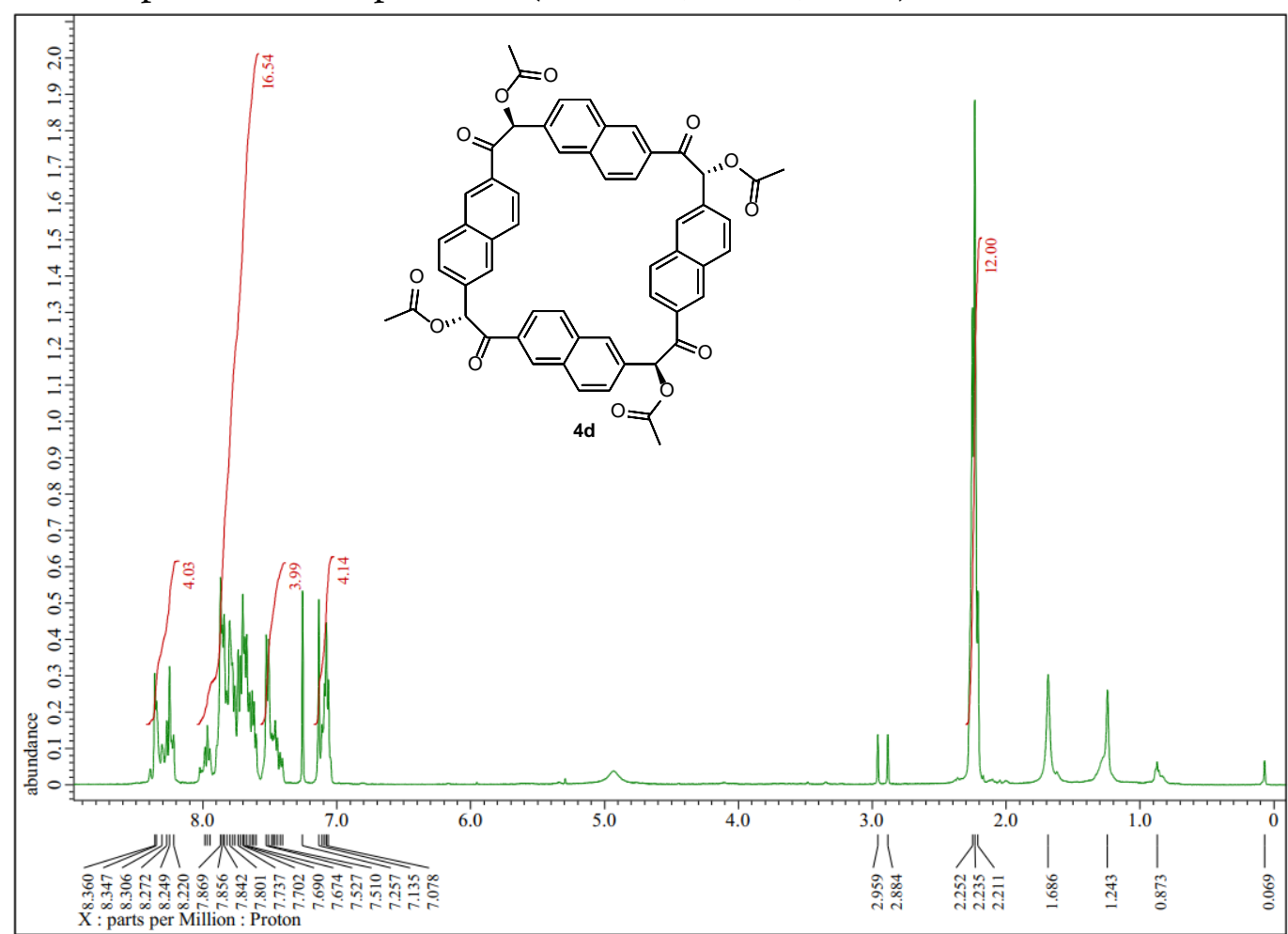

Figure S1 1. ${ }^{13} \mathrm{C}$ Spectrum of compound $4 \mathbf{d}\left(125 \mathrm{MHz}, \mathrm{CDCl}_{3}, 25^{\circ} \mathrm{C}\right)$.

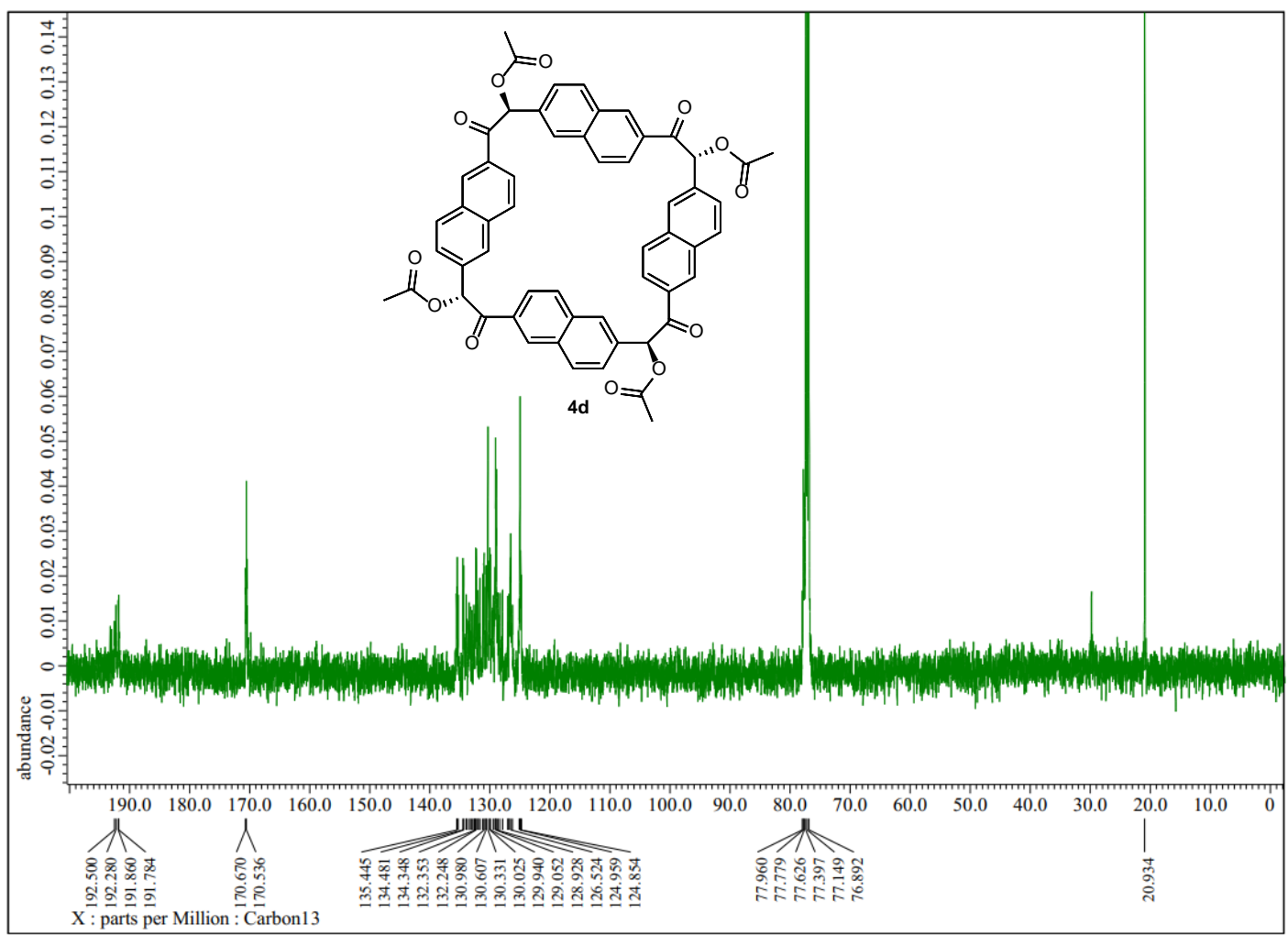


Figure S12. ${ }^{1} \mathrm{H}$ Spectrum of compound $\mathbf{5 d}\left(500 \mathrm{MHz}, \mathrm{CDCl}_{3}, 25^{\circ} \mathrm{C}\right)$.

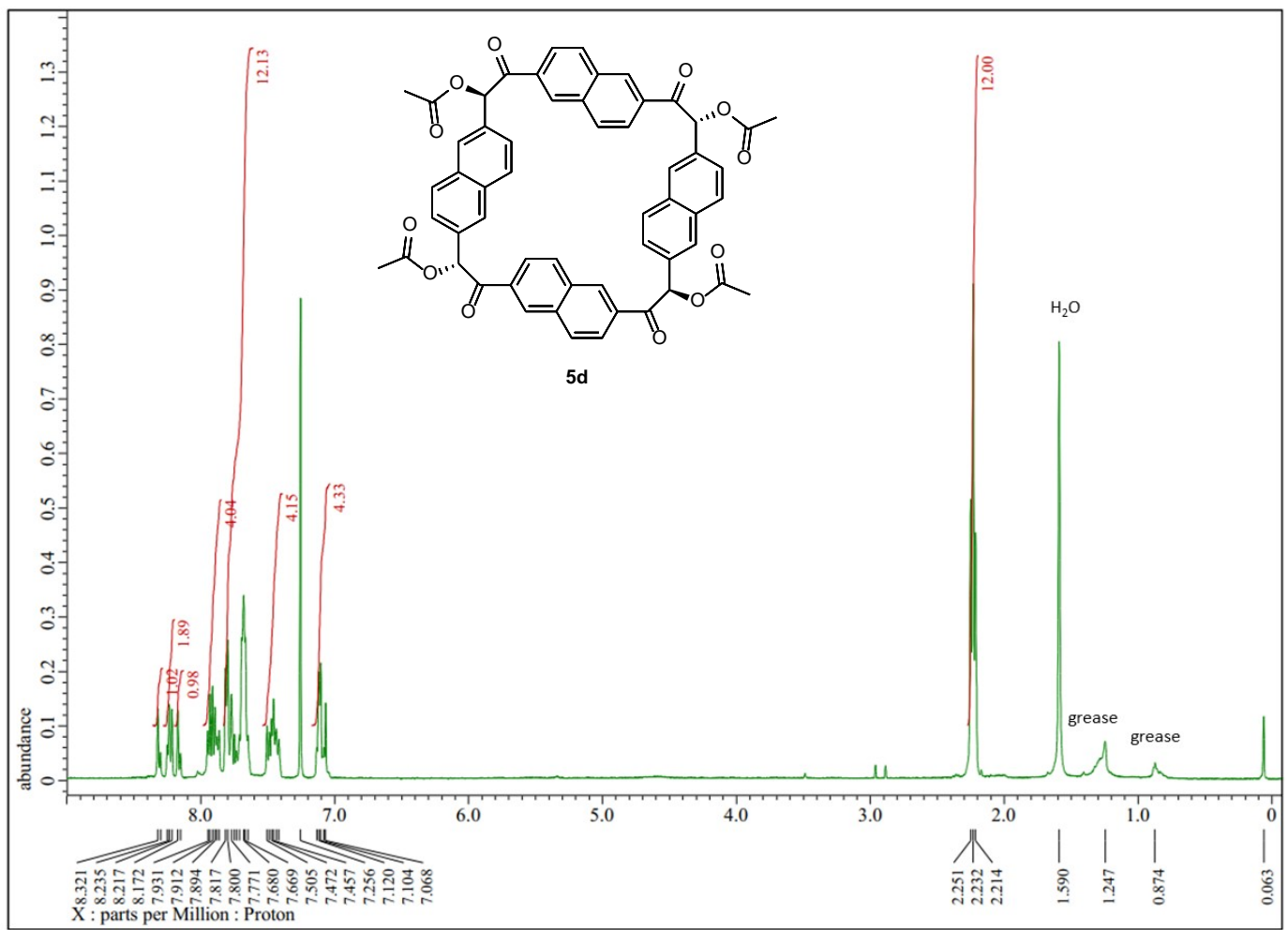

Figure S13. ${ }^{13} \mathrm{C}$ Spectrum of compound $\mathbf{5 d}\left(125 \mathrm{MHz}, \mathrm{CDCl}_{3}, 25^{\circ} \mathrm{C}\right)$.

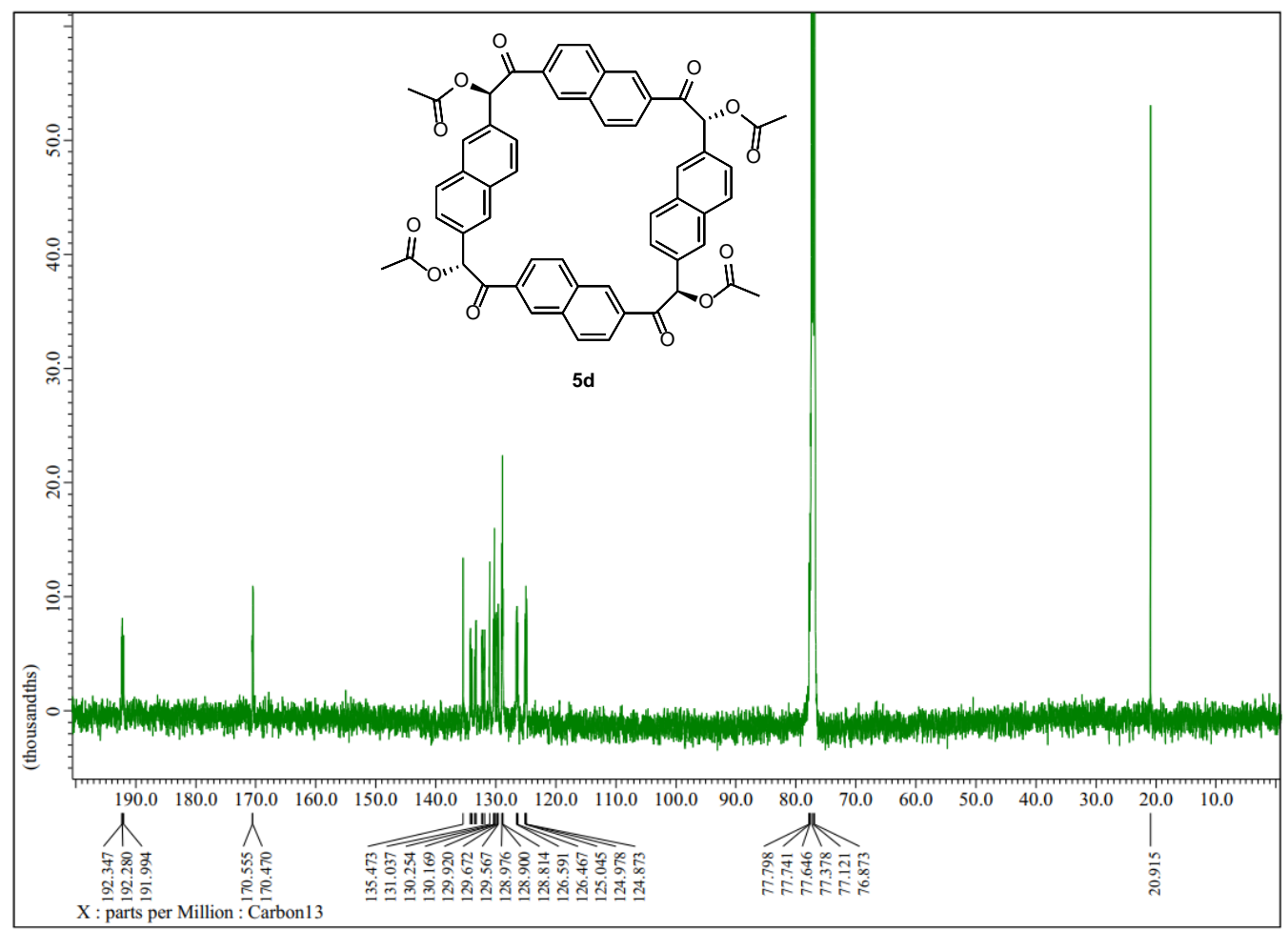




\section{${ }^{1} \mathrm{H},{ }^{13} \mathrm{C}$ NMR, IR, and HR-ESI Mass Spectra of Complex 6}

Figure S14. ${ }^{1} \mathrm{H}$ NMR Spectrum of compound $6\left(500 \mathrm{MHz}, \mathrm{CDCl}_{3}, 25^{\circ} \mathrm{C}\right)$.

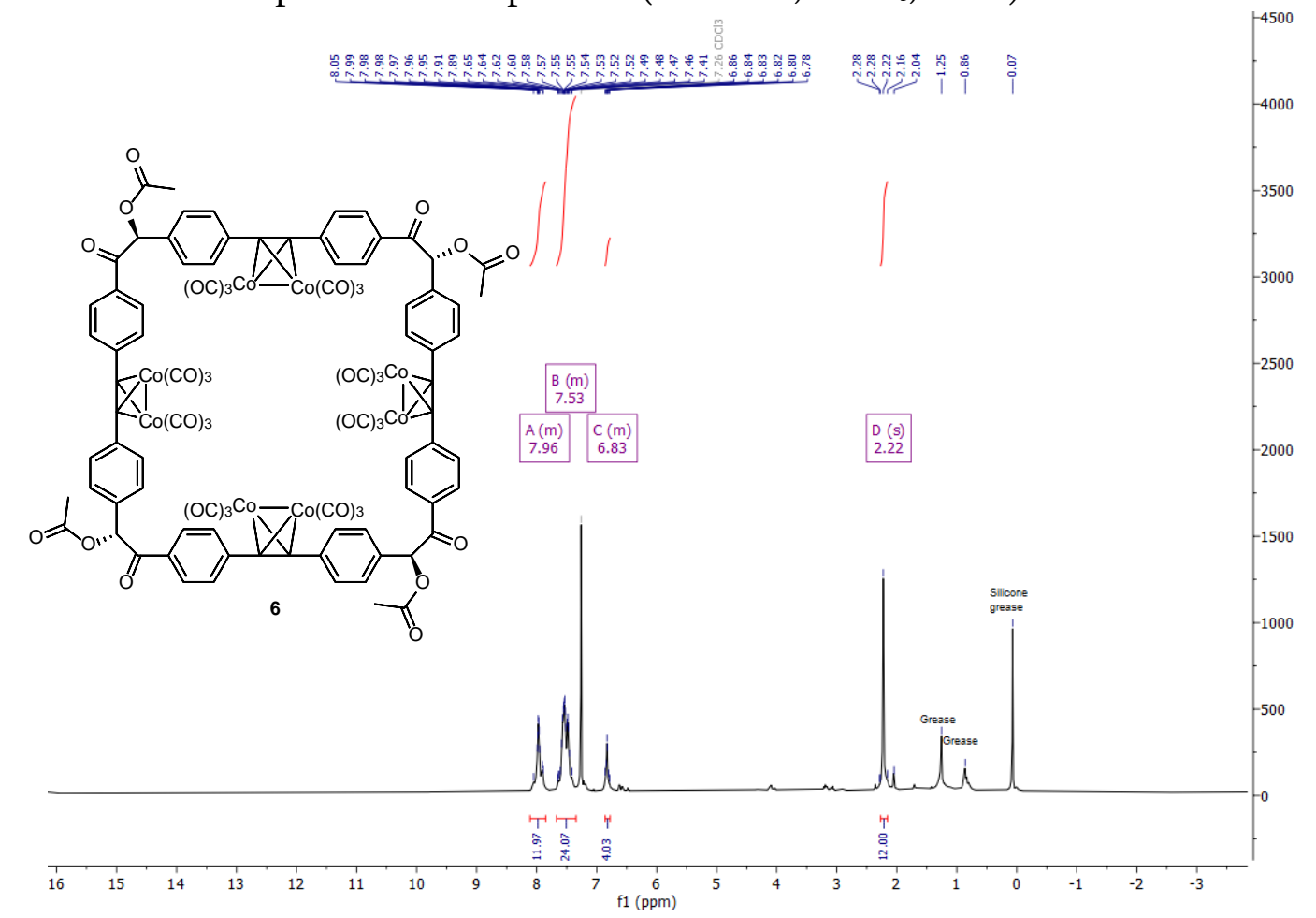

Figure S15. ${ }^{13} \mathrm{C}$ NMR Spectrum of compound $6\left(125 \mathrm{MHz}, \mathrm{CDCl}_{3}, 25{ }^{\circ} \mathrm{C}\right)$.

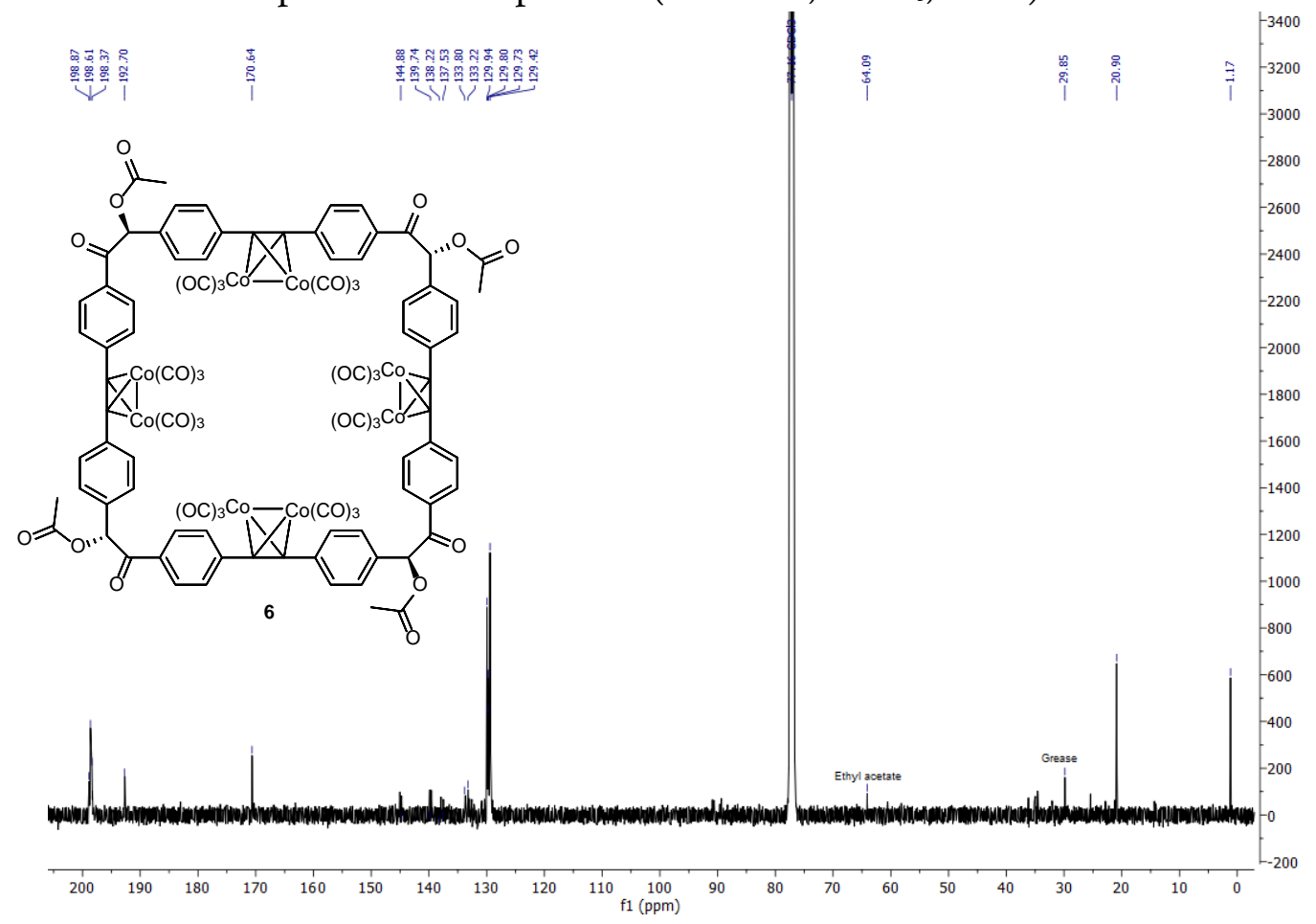


Figure S16. Comparison of the IR spectra of compounds $4 c / 5 c$ and 6 .

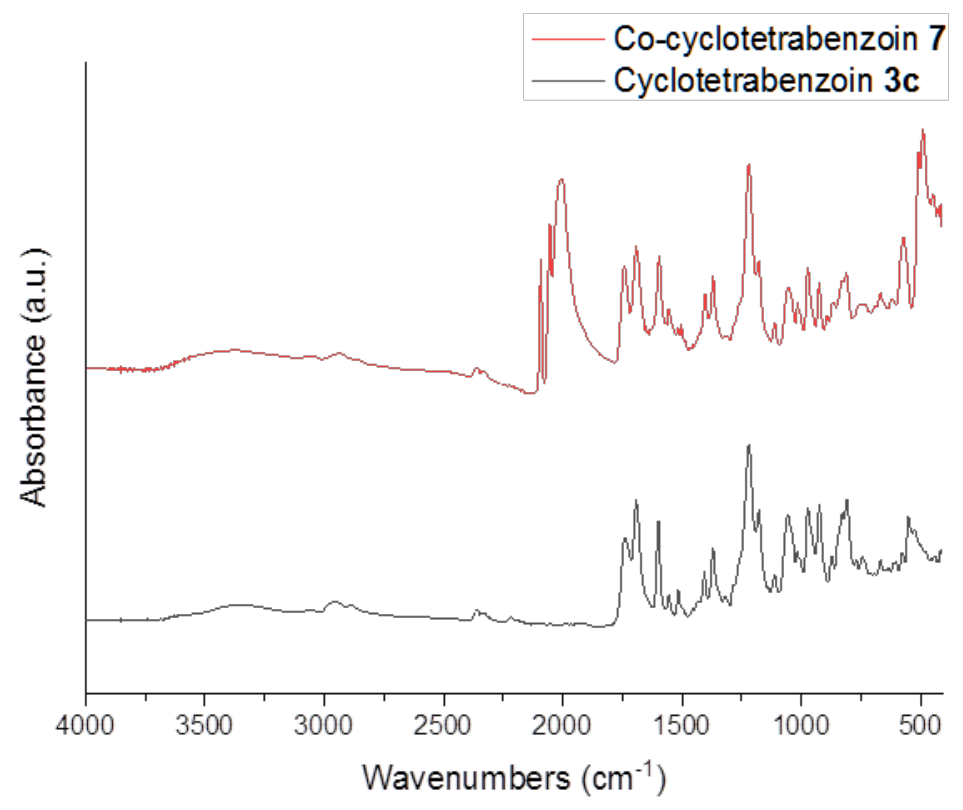

Figure S17. Comparison of the UV-vis spectra of compounds $4 \mathbf{c} / \mathbf{5 c}$ and $\mathbf{6}(8.9 \mu \mathrm{M}$ solutions in $\mathrm{CH}_{2} \mathrm{Cl}_{2}$ ).

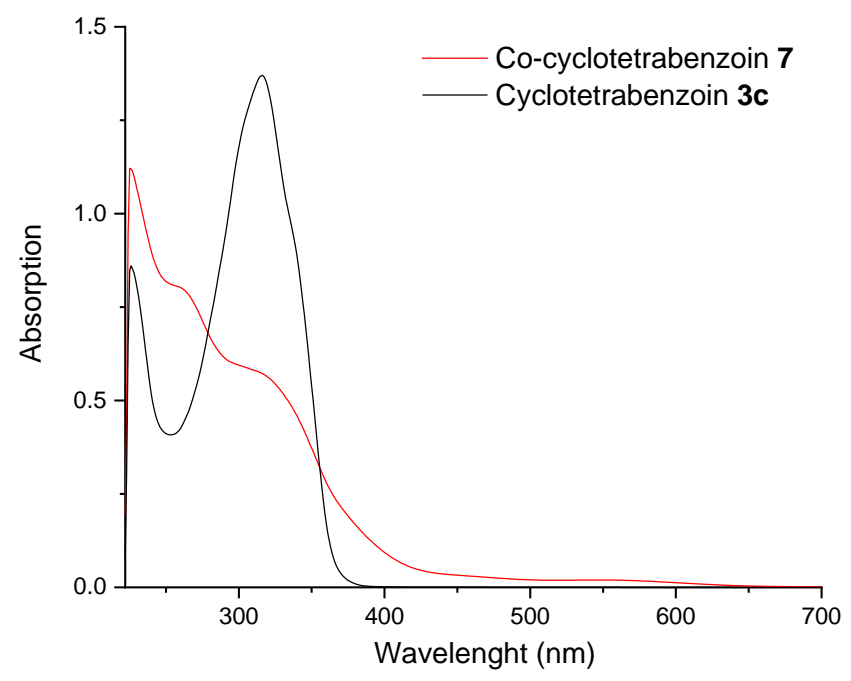


Figure S18. Comparison of ${ }^{1} \mathrm{H}$ NMR spectra of compounds $4 \mathrm{c} / 5 \mathrm{c}$ and 6

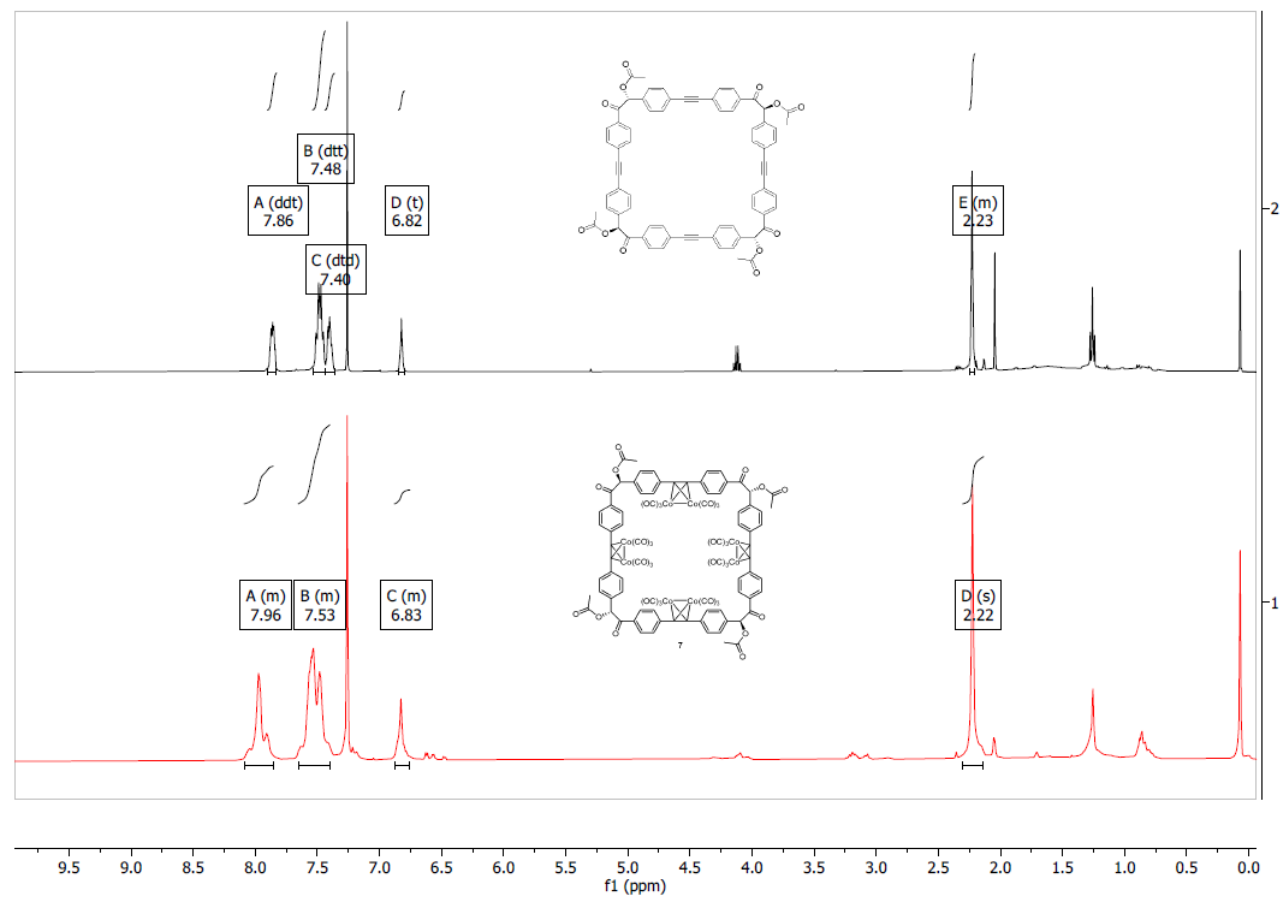

Figure S19. Comparison of ${ }^{13} \mathrm{C}$ NMR spectra of compounds $4 c / 5 c$ and 6

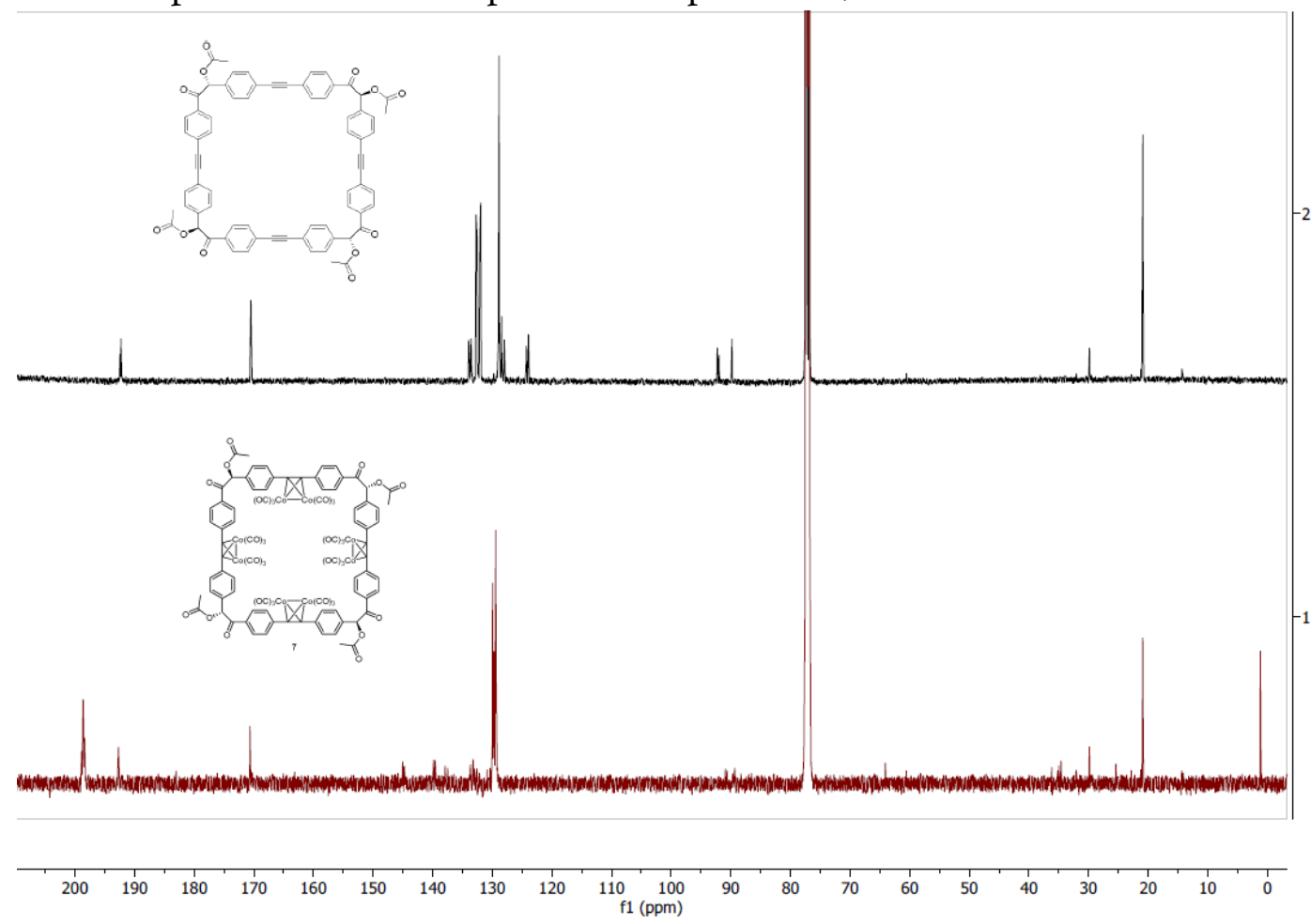


Figure S20. HR-ESI MS spectra of compound 6.
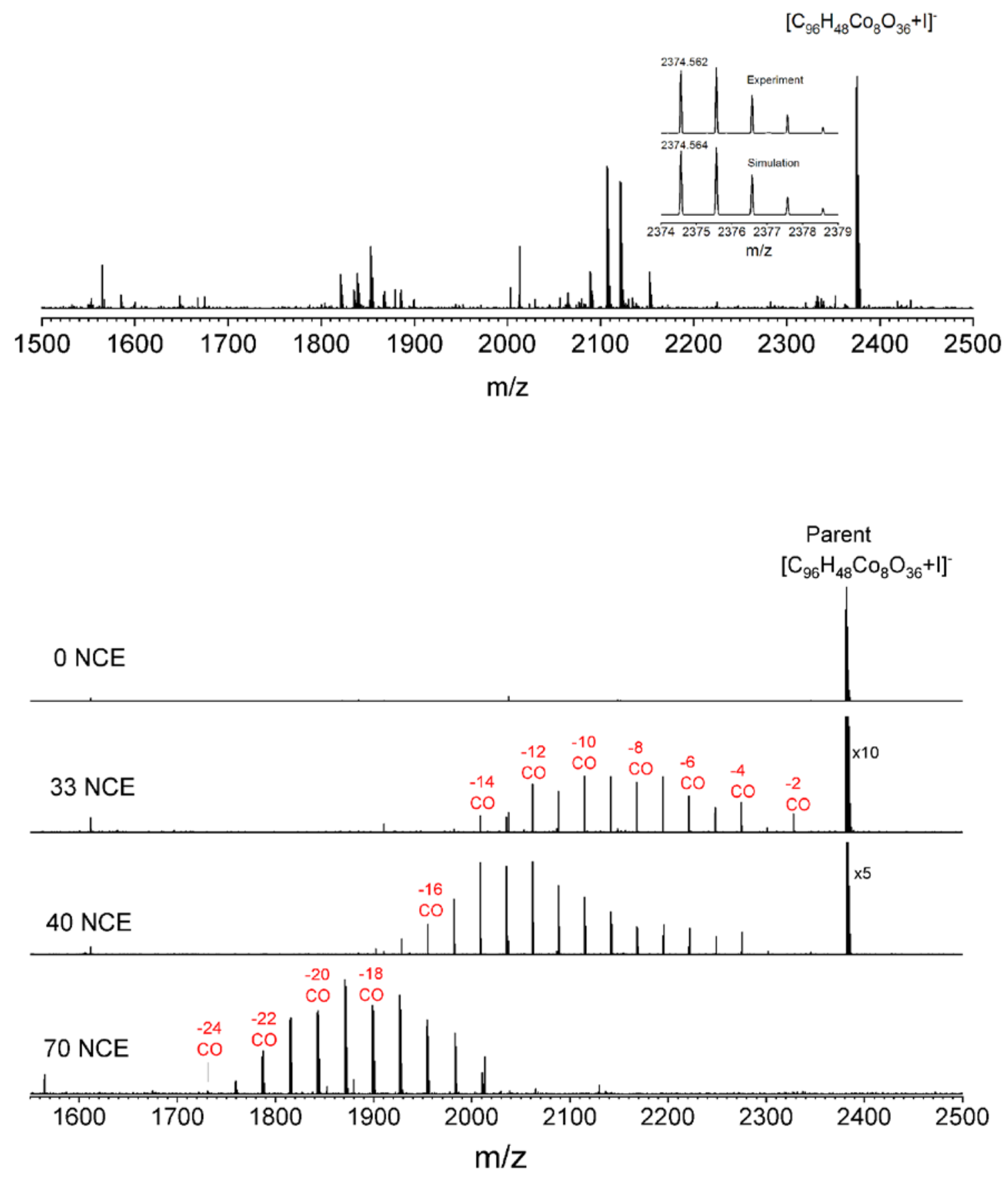

Top trace: ESI-MS of $\mathbf{6}$ as iodide adduct, negative mode.

Bottom traces: Collision-induced dissociation of $[6+\mathrm{I}]^{-}$at four different activation energies (in machine units of "normalized collision energy", NCE) indicating loss of up to $24 \mathrm{CO}$ molecules. 


\section{References}

(1) Xie, G.-Y.; Jiang, L.; Lu, T.-B. Dalton Trans. 2013, 42, 14092-14099.

(2) Ji, Q.; Le, H. T. M.; Wang, X.; Chen, Y.-S.; Makarenko, T.; Jacobson, A. J.; Miljanić, O. Š. Chem. Eur. J. 2015, 21, 17205-17209.

(3) Shin, W. K.; Kang, D.; An, D. K. Bull. Kor. Chem. Soc. 2014, 35, 2169-2171.

(4) Burrows, A. D.; Frost, C. G.; Mahon, M. F.; Richardson, C. Chem. Commun. 2009, 28, 42184220.

(5) Helms, A.; Heiler, D.; McLendon, G. J. Am. Chem. Soc. 1992, 114, 6227-6238.

(6) Wulff, G.; Lauer, M.; Disse, B. Chem. Ber./Recl. 1979, 112, 2854-2865.

(7) Bondarenko, L.; Dix, I.; Hinrichs, H.; Hopf, H. Synthesis 2004, 2751-2759.

(8) Dix, I.; Hopf, H.; Satyanarayana, T. B. N.; Ernst, L. Beilstein J. Org. Chem. 2010, 6, 932-937. 